\title{
COMPLETE INTERPOLATING SEQUENCES, THE DISCRETE MUCKENHOUPT CONDITION, AND CONFORMAL MAPPING
}

\author{
Gunter Semmler \\ TU Bergakademie Freiberg, Institut für Angewandte Analysis \\ Prüferstraße 9, 09596 Freiberg, Germany; semmler@math.tu-freiberg.de
}

\begin{abstract}
We extend the parameterization of sine-type functions in terms of conformal mappings onto slit domains given by Eremenko and Sodin to the more general case of generating functions of real complete interpolating sequences. It turns out that the cuts have to fulfill the discrete Muckenhoupt condition studied earlier by Lyubarskii and Seip.
\end{abstract}

\section{Interpolation in Paley-Wiener spaces and Riesz bases of exponentials}

Let $P W_{\pi}^{2}$ be the Paley-Wiener space of all entire functions of exponential type at most $\pi$ which belong to $L^{2}$ on the real line. A sequence $\left\{\lambda_{n}\right\}_{n \in \mathbf{Z}}$ of complex numbers is called a complete interpolating sequence if for all complex sequences $\left\{a_{n}\right\}_{n \in \mathbf{Z}}$ with

$$
\sum_{n \in \mathbf{Z}}\left|a_{n}\right|^{2} \mathrm{e}^{-2 \pi\left|\operatorname{Im} \lambda_{n}\right|}\left(1+\left|\operatorname{Im} \lambda_{n}\right|\right)<\infty
$$

the interpolation problem

$$
f\left(\lambda_{n}\right)=a_{n}
$$

has a unique solution $f \in P W_{\pi}^{2}$. It is known (see [22]) that for complete interpolating sequences the function $f$ depends continuously on the sequence $\left\{a_{n}\right\}$ in the sense that there are constants $C, c>0$ independent of $\left\{a_{n}\right\}$ such that

$$
c\|f\|_{L^{2}(\mathbf{R})}^{2} \leq \sum_{n \in \mathbf{Z}}\left|a_{n}\right|^{2} \mathrm{e}^{-2 \pi\left|\operatorname{Im} \lambda_{n}\right|}\left(1+\left|\operatorname{Im} \lambda_{n}\right|\right) \leq C\|f\|_{L^{2}(\mathbf{R})}^{2} .
$$

If $\left\{\lambda_{n}\right\} \subset \mathbf{R}$ condition (1) just means that $\left\{a_{n}\right\} \in l^{2}(\mathbf{Z})$, and in the sequel we will only consider this case.

A sequence $\left\{e_{n}\right\}$ of vectors in a Hilbert space $H$ is called a Riesz basis in $H$ if it is complete and there are $C, c>0$ such that

$$
c \sum\left|a_{n}\right|^{2} \leq\left\|\sum a_{n} e_{n}\right\|^{2} \leq C \sum\left|a_{n}\right|^{2}
$$

holds for every finite sequence $\left\{a_{n}\right\}$ of numbers. See [4] for basic information on this topic and equivalent definitions. Using the fact that the Fourier transform provides an isometry between $L^{2}(-\pi, \pi)$ and $P W_{\pi}^{2}$, one can characterize complete interpolating sequences as those sequences for which the set $\left\{\mathrm{e}^{\lambda_{n} i t}\right\}_{n \in \mathbf{Z}}$ is a Riesz basis for $L^{2}(-\pi, \pi)$, cf. Theorem 9 in chapter 4 of [34].

doi:10.5186/aasfm.2010.3502

2000 Mathematics Subject Classification: Primary 42A65, 30D15, 30C20.

Key words: Complete interpolating sequences, nonharmonic Fourier series, Riesz basis of exponentials, generating function, interpolation in Paley-Wiener spaces, discrete Muckenhoupt condition, sine-type function. 
It is also well-known that a sequence $\left\{e_{n}\right\}$ of vectors in a Hilbert space $H$ is a Riesz basis in $H$ if and only if it is an unconditional basis with $0<\inf _{n}\left\|e_{n}\right\| \leq \sup _{n}\left\|e_{n}\right\|<$ $\infty$, see [9],[26]. Therefore, some of our cited references deal with unconditional bases of exponentials.

The problem to describe all complete interpolating sequences has been studied since the classical book [25] of Paley and Wiener. The first type of results in this direction were perturbation statements of the set of integers, and we provide as an example the famous theorem of Kadets [13], see also [34].

Theorem 1. A sequence $\left\{\lambda_{n}\right\}_{n \in \mathbf{Z}}$ of real numbers is a complete interpolating sequence if

$$
\sup _{n \in \mathbf{Z}}\left|\lambda_{n}-n\right|<\frac{1}{4}
$$

The constant $\frac{1}{4}$ cannot be replaced by any larger number.

A full description of all complete interpolating sequences on the real line was obtained by Pavlov [27], who proved the following theorem.

Theorem 2. A sequence $\left\{\lambda_{n}\right\}_{n \in \mathbf{Z}}$ of real numbers is a complete interpolating sequence if and only if

(i) it is separated, i.e.

$$
\delta:=\inf _{n \neq m}\left|\lambda_{n}-\lambda_{m}\right|>0
$$

(ii) the limit ${ }^{1}$

$$
F(z)=\lim _{R \rightarrow \infty} \prod_{\left|\lambda_{n}\right|<R}\left(1-\frac{z}{\lambda_{n}}\right)
$$

exists uniformly on compact subsets of $\mathbf{C}$ and defines an entire function $F$ of exponential type $\pi$, the generating function,

(iii) the function $w(x):=|F(x+i y)|^{2}, x \in \mathbf{R}$, satisfies the Muckenhoupt condition

$$
\int_{I} w(x) d x \int_{I} \frac{1}{w(x)} d x \leq C|I|^{2}
$$

for some constant $C>0$, some $y \neq 0$ (and hence for all $y \neq 0$ ), and all intervals $I \subset \mathbf{R}$ of finite length $|I|$.

This formulation of the theorem is specialized for real $\lambda_{n}$, whereas [27] contains conditions even if the $\lambda_{n}$ are in some horizontal strip. Later this restriction was completely disposed of by Nikolskii [24] and Minkin [23].

Because of the separation condition (3) we can in the following always assume that the $\left\{\lambda_{n}\right\}$ are ordered, i.e. $\lambda_{n+1}>\lambda_{n}$ for all $n \in \mathbf{Z}$. Besides, it is also well-known (cf. [22]) that a (real) complete interpolating sequence is relatively dense, i.e. for some $\varepsilon>0$ the sets $\left\{\lambda_{n}\right\}_{n \in \mathbf{Z}} \cap[x-\varepsilon, x+\varepsilon]$ are nonempty for every $x \in \mathbf{R}$. In particular,

$$
\Delta:=\sup _{n \in \mathbf{Z}}\left|\lambda_{n+1}-\lambda_{n}\right|<\infty
$$

\footnotetext{
${ }^{1}$ If one of the numbers $\lambda_{n}$ is equal to zero, the corresponding factor has to be replaced by $z$. This convention will also be adopted in what follows.
} 
Theorem 2 has been deduced in a different manner and generalized by Sedletskii [31], [32] and Lyubarskii and Seip [22]. In the latter paper it is shown that condition (iii) in this theorem can be replaced by

(iii') There is a relatively dense subsequence $\left\{\lambda_{n_{k}}\right\}_{k \in \mathbf{Z}}$ such that the numbers $d_{k}:=$ $\left|F^{\prime}\left(\lambda_{n_{k}}\right)\right|^{2}$ satisfy the discrete Muckenhoupt condition

$$
\sum_{n \in I} d_{n} \sum_{n \in I} d_{n}^{-1} \leq C|I|^{2}
$$

for a constant $C>0$ and all finite sets $I$ of consecutive integers containing $|I|$ elements.

Checking the Muckenhoupt condition $\left(A_{2}\right)$ for a function $F$ given by an infinite product (converging in the Cauchy principal value sense) is practically quite hard. Condition $\left(\tilde{A}_{2}\right)$ is already easier to verify since it involves only countably many sets $I$ instead of all finite intervals.

The goal of this paper is to give an alternative characterization of complete interpolating sequences. In contrast to existing characterizations our result can be regarded as a parameterization of the set of complete interpolating sequences by independent parameters. Once this is done we can easily give discrete analogues of well-known sufficient conditions for such sequences. As a by-product we obtain a different way to represent the generating function. In order to motivate our construction we revise in the next section the concept of sine-type functions.

\section{Sine-type functions}

An entire function $F$ of exponential type is called a sine-type function (of type $\sigma)$ if

(i) $F$ has exponential type $\sigma(0<\sigma<\infty)$ in each of the half-planes $\mathbf{C}_{+}:=$ $\{\operatorname{Im} z>0\}$ and $\mathbf{C}_{-}:=\{\operatorname{Im} z<0\}$

(ii) the zeros $\left\{\lambda_{n}\right\}_{n \in \mathbf{Z}}$ of $F$ are located in some strip $\{|\operatorname{Im} z| \leq h\}$ and satisfy the separation condition (3),

(iii) for some $y \neq 0, C, c>0$ and all $x \in \mathbf{R}$ we have $c \leq|f(x+\mathrm{i} y)| \leq C$.

This definition goes back to Levin [17], [18]. Together with Golovin [11] he proved

Theorem 3. The zeros $\left\{\lambda_{n}\right\}_{n \in \mathbf{Z}}$ of a function of sine-type $\pi$ form a complete interpolating sequence.

An exposition of this result and some further developments can be found in [19] and [34]. Though not every complete interpolating sequence coincides with the zeros of some sine-type function, the introduction of this notion was not too far away from the exhaustive characterization in Theorem 2, as the following theorem of Avdonin and Joó [3] shows.

Theorem 4. If $\left\{\lambda_{n}\right\}_{n \in \mathbf{Z}} \subset \mathbf{R}$ is a complete interpolating sequence then there is $d \in\left(0, \frac{1}{4}\right)$ and a function $F$ of sine-type $\pi$ with zeros $\left\{\mu_{n}\right\}_{n \in \mathbf{Z}}$ such that

$$
d\left(\lambda_{n-1}-\lambda_{n}\right) \leq \mu_{n}-\lambda_{n} \leq d\left(\lambda_{n+1}-\lambda_{n}\right)
$$

for all $n \in \mathbf{Z}$.

As noticed by Levin and Ostrovskii [21], a parametric description of all sine-type functions is desirable, and this problem was completely solved by Eremenko and Sodin [6]. Since their construction will be of importance to us later, we describe it in some 
detail. First we recall that Levin and Ostrovskii [20] proved that a sequence $\left\{\lambda_{n}\right\}_{n \in \mathbf{Z}}$ with $\left|\operatorname{Im} \lambda_{n}\right| \leq h$ is the zero sequence of a sine-type function if and only if $\left\{\operatorname{Re} \lambda_{n}\right\}_{n \in \mathbf{Z}}$ is so. We can therefore restrict our considerations to sine-type functions $F$ with real zeros. Such functions belong to the Laguerre-Pólya class $\mathscr{L} \mathscr{P}$ of real entire functions approximable by real polynomials with real zeros uniformly on every compact subset of $\mathbf{C}$. We may number the critical points of a function $F \in \mathscr{L} \mathscr{P}$ in increasing order and counting multiplicities, so that the indices of all points of maximum are even. Let $c_{n}$ be the value of $F$ at the $n$-th critical point and denote by $\operatorname{Cr} F:=$ $\left\{\ldots, c_{-1}, c_{0}, c_{1}, \ldots\right\}$ the set of all critical values. If $\mathrm{Cr} F$ is finite from the left (from the right) and $c_{m}$ is its first (last) member then we set $c_{m-1}=\lim _{x \rightarrow-\infty} F(x)\left(c_{m+1}=\right.$ $\left.\lim _{x \rightarrow+\infty} F(x)\right)$, which is allowed to be infinite. The sequence $\mathrm{Cr} F$ is alternating, i.e. $(-1)^{n} c_{n} \geq 0$.

For an arbitrary alternating sequence $s=\left\{c_{n}\right\}$ a function $F \in \mathscr{L} \mathscr{P}$ with $\operatorname{Cr} F=s$ can be constructed in the following way. Define a simply connected comb-like domain

$$
\Omega(s):=\mathbf{C} \backslash \bigcup_{n}\left\{z=x+\mathrm{i} n \pi:-\infty<x \leq \log \left|c_{n}\right|\right\} .
$$

If there is an infinite first (last) member $c_{m}$ of the sequence $s$ we consider the domain lying in the half plane $\{\operatorname{Im} z>(m-1) \pi\}(\{\operatorname{Im} z<(m+1) \pi\})$. Let $\varphi: \mathbf{C}_{-} \rightarrow \Omega(s)$ be a conformal map of the lower half-plane onto $\Omega(s)$ such that $\operatorname{Re} \varphi(\mathrm{i} y) \rightarrow \infty$ as $y \rightarrow-\infty$. Then the function

$$
F(z)=\mathrm{e}^{\varphi(z)}
$$

can be extended to an entire function by the Schwarz reflection principle and it can be shown that $F \in \mathscr{L} \mathscr{P}$ and $\mathrm{Cr} F=s$. Conversely, every function $F \in \mathscr{L} \mathscr{P}$ has the representation (6) with $\varphi$ a conformal map of $\mathbf{C}_{-}$onto $\Omega(\mathrm{Cr} F)$. Eremenko and Sodin [6] specify now conditions on the sequence $\left\{c_{n}\right\}$ in order that the function (6) is of sine-type.

Theorem 5. A real entire function $F$ is of sine-type if and only if it can be represented in the form (6) where $\varphi: \mathbf{C}_{-} \rightarrow \Omega(s)$ is a conformal map with $\operatorname{Re} \varphi(\mathrm{i} y) \rightarrow$ $\infty$ as $y \rightarrow-\infty$, and the sequence $s=\left\{c_{n}\right\}_{n \in \mathbf{Z}}=\operatorname{Cr} F$ satisfies

$$
c \leq\left|c_{n}\right| \leq C \quad \forall n \in \mathbf{Z}
$$

with some constants $C, c>0$.

In fact, [6] contains a more general statement that even characterizes sine-type functions where the separability condition (3) in the definition is omitted, but we do not need this since in view of condition (i) in Theorem 2 we will only be concerned with separated sequences. Parameterizations of classes of entire functions by conformal mappings onto certain slit domains have a long history connected with the names of MacLane, Vinberg, Marchenko, and Ostrovskii, see the references in [6].

\section{Characterization of complete interpolating sequences}

For any real complete interpolating sequence $\left\{\lambda_{n}\right\}_{n \in \mathbf{Z}}$ the generating function (4) belongs to the Laguerre-Pólya class $\mathscr{L} \mathscr{P}$ and has therefore a representation (6) with a conformal map $\varphi: \mathbf{C}_{-} \rightarrow \Omega(s)$. It is therefore natural to ask for conditions on the sequence $s=\left\{c_{n}\right\}$ that characterize real complete interpolating sequences. Clearly, such conditions must be more general than (7). 
The crucial point is the Muckenhoupt condition $\left(A_{2}\right)$, and in order to develop an idea we remark that any finite interval $I$ can be decomposed into subintervals say $I_{p}, \ldots, I_{q}$ such that the segment $I_{n}+\mathrm{i} y(y<0$ fixed $)$ is mapped by $\varphi$ near the $n$-th endpoint of the cuts of $\Omega(s)$, i.e.

$$
\varphi(x+\mathrm{i} y) \approx \log \left|c_{n}\right|+\mathrm{i} n \pi, \quad x \in I_{n}, n=p, \ldots, q,
$$

see Figure 1.

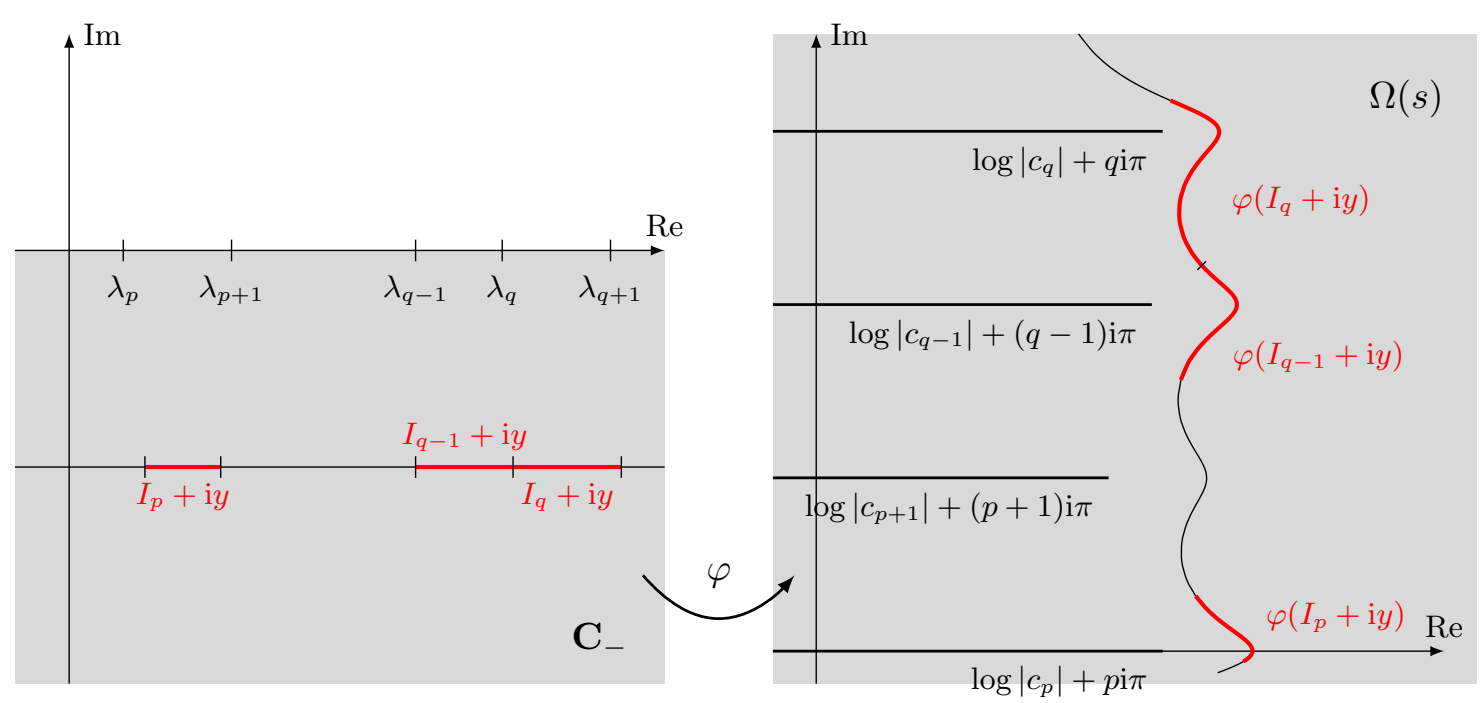

Figure 1. Conformal mapping of $\mathbf{C}_{-}$onto $\Omega(s)$.

If we approximate the occurring integrals by its Riemann sums we get

$$
\int_{I}|F(x+\mathrm{i} y)|^{2} d x \approx \sum_{n=p}^{q} c_{n}^{2}\left|I_{n}\right|, \quad \int_{I}|F(x+\mathrm{i} y)|^{-2} d x \approx \sum_{n=p}^{q} c_{n}^{-2}\left|I_{n}\right| .
$$

In view of properties (3), (5) we can expect that also

$$
\delta^{\prime} \leq\left|I_{n}\right| \leq \Delta^{\prime}, \quad n=p, \ldots, q,
$$

for some constants $\delta^{\prime}, \Delta^{\prime}>0$ that do not depend on $I$. Hence it turns out that it will just be the discrete Muckenhoupt condition $\left(\tilde{A}_{2}\right)$ for the numbers $d_{n}=c_{n}^{2}$ that is our desired characterization. Making these informal arguments rigorous is enough for showing the following statement.

Theorem 6. Let $s=\left\{c_{n}\right\}_{n \in \mathbf{Z}}$ be a sequence with $(-1)^{n} c_{n} \geq 0$, and let $\left\{d_{n}\right\}_{n \in \mathbf{Z}}=$ $\left\{c_{n}^{2}\right\}_{n \in \mathbf{Z}}$ satisfy the discrete Muckenhoupt condition $\left(\tilde{A}_{2}\right)$. Then for every conformal $\operatorname{map} \varphi: \mathbf{C}_{-} \rightarrow \Omega(s)$ with $\lim _{y \rightarrow-\infty} \operatorname{Re} \varphi(\mathrm{i} y)=\infty$ the function $F$ in (6) is an entire function of exponential type. $\varphi$ can be normalized so that the exponential type of $F$ is $\pi$, and in this case the zero set $\left\{\lambda_{n}\right\}_{n \in \mathbf{Z}} \subset \mathbf{R}$ of $F$ is a complete interpolating sequence. Conversely, every complete interpolating sequence is obtained in this way.

Proof. It suffices to adapt arguments from [6], which make use of ideas from [10]. For the reader's convenience we provide the details. In order to structure the proof we formulate important steps as lemmas.

First suppose that the sequence $\left\{d_{n}\right\}_{n \in \mathbf{Z}}=\left\{c_{n}^{2}\right\}_{n \in \mathbf{Z}}$ satisfies the discrete Muckenhoupt condition $\left(\tilde{A}_{2}\right)$, and $\varphi: \mathbf{C}_{-} \rightarrow \Omega(s)$ is a conformal map with $\lim _{y \rightarrow-\infty} \operatorname{Re} \varphi(\mathrm{i} y)$ 
$=\infty$. Choosing $I=\{p, p+1, \ldots, q\}$ in $\left(\tilde{A}_{2}\right)$ we obtain

$$
c_{p}^{2} c_{q}^{-2} \leq \sum_{n=p}^{q} c_{n}^{2} \sum_{n=p}^{q} c_{n}^{-2} \leq C(q-p+1)^{2} .
$$

Hence for some $C_{1}>0$ we have

$$
|\log | c_{p}|-\log | c_{q}|| \leq C_{1}+\log |p-q| \quad \forall p \neq q,
$$

and in particular

$$
-C_{2}-\log |n| \leq \log \left|c_{n}\right| \leq C_{2}+\log |n| \quad \forall n \in \mathbf{Z} \backslash\{0\},
$$

where $C_{2}:=\log \left|c_{0}\right|+C_{1}$. For $r>|\log | c_{0}||$ let $L(r)$ be the connected arc of $\Omega(s) \cap$ $\{|z|=r\}$ that intersects the positive real axis and let $l(r)$ denote its length. Next we show that the preimage of $L(r)$ is almost a semicircle in $\mathbf{C}_{-}$.

Lemma 1. For $r \rightarrow \infty$ the relations

$$
\sup _{z \in L(r)}\left|\varphi^{-1}(z)\right|=A(1+o(1)) r \quad \text { and } \quad \inf _{z \in L(r)}\left|\varphi^{-1}(z)\right|=A(1+o(1)) r
$$

hold with a constant $A>0$.

Proof. Let $\Omega\left(r_{1}, r_{2}\right) \quad\left(0<r_{1}<r_{2}\right)$ be the component of $\Omega(s) \backslash\left(L\left(r_{1}\right) \cup L\left(r_{2}\right)\right)$ which contains $\frac{1}{2}\left(r_{1}+r_{2}\right)$ and $M\left(r_{1}, r_{2}\right):=\bmod \Gamma_{r_{1}, r_{2}}$ be the module of the family $\Gamma_{r_{1}, r_{2}}$ of curves in $\Omega\left(r_{1}, r_{2}\right)$ separating $L\left(r_{1}\right)$ and $L\left(r_{2}\right)$. The paper [10] provides the following consequence of one of Teichmüller's module theorems: If $\Phi(r), r \geq r_{0}$, is a function such that

$$
\lim _{r_{1}, r_{2} \rightarrow \infty} M\left(r_{1}, r_{2}\right)-\left(\Phi\left(r_{2}\right)-\Phi\left(r_{1}\right)\right)=0
$$

then there is $A>0$ such that

$$
\sup _{z \in L(r)}\left|\varphi^{-1}(z)\right|=(1+o(1)) A \mathrm{e}^{\pi \Phi(r)} \quad \text { and } \inf _{z \in L(r)}\left|\varphi^{-1}(z)\right|=(1+o(1)) A \mathrm{e}^{\pi \Phi(r)} .
$$

Applying this theorem for $\Phi(r)=\frac{1}{\pi} \log r$, we obtain the desired conclusion.

Let $a(r), b(r)$ be the endpoints of $L(r)$. Then $a(r)=x(r)+\mathrm{i} \pi n(r)$ for some $n=n(r)>0$, and $x=x(r) \leq \log \left|c_{n}\right|$. If $x(r) \leq 0$ then $\log \left|c_{n+1}\right|+\mathrm{i} \pi(n+1)$ lies outside the circle of radius $r$ centered at the origin, hence

$$
\left(\log \left|c_{n+1}\right|\right)^{2}+\pi^{2}(n+1)^{2}>r^{2}=x^{2}+\pi^{2} n^{2}
$$

and (9) yields

$$
\left(C_{2}+\log (n+1)\right)^{2}+(2 n+1) \pi^{2}>x^{2}
$$

and therefore with $r \geq \pi n$

$$
-x(r) \leq C_{3} \sqrt{n} \leq \pi^{-\frac{1}{2}} C_{3} \sqrt{r}
$$

with some constant $C_{3}>0$ independent of $n$ and $r$. For $x(r) \geq 0$ we apply (9) to obtain

$$
x(r) \leq \log \left|c_{n}\right| \leq C_{2}+\log n \leq C_{2}-\log \pi+\log r .
$$

The analogous estimate

$$
-\pi^{-\frac{1}{2}} C_{3} \sqrt{r} \leq \operatorname{Re} b(r) \leq C_{2}-\log \pi+\log r
$$


is valid for the other endpoint. Hence we find for the length of $L(r)$

$$
\pi r-2 r \arcsin \left(\frac{C_{2}-\log \pi+\log r}{r}\right) \leq l(r) \leq \pi r+2 r \arcsin \left(\frac{\pi^{-\frac{1}{2}} C_{3} \sqrt{r}}{r}\right),
$$

and therefore

$$
\pi\left(r-C_{4} \log r\right) \leq l(r) \leq \pi\left(r+C_{4} \sqrt{r}\right)
$$

with a constant $C_{4}>0$. By elementary properties of the module (cf. [1], Chapter 4), $M\left(r_{1}, r_{2}\right)$ is bounded from above by the module $s\left(r_{1}, r_{2}\right)$ of the family of curves joining the noncircular sides of

$$
S\left(r_{1}, r_{2}\right):=\left\{z \in \mathbf{C}: r_{1}<|z|<r_{2},|\arg z|<\frac{\pi}{2}\left(r-C_{4} \log r\right) / r\right\}
$$

$$
M\left(r_{1}, r_{2}\right) \leq s\left(r_{1}, r_{2}\right)
$$

We postpone the proof that

$$
\left|s\left(r_{1}, r_{2}\right)-\frac{1}{\pi} \log \frac{r_{2}}{r_{1}}\right| \rightarrow 0 \quad \text { as } \quad r_{1}, r_{2} \rightarrow \infty
$$

to Lemma 7. A lower estimate of the module follows from the Grötzsch principle (cf. [28], Proposition 11.12)

$$
M\left(r_{1}, r_{2}\right) \geq \int_{r_{1}}^{r_{2}} \frac{d r}{l(r)}
$$

With the help of (14) we find

(18) $M\left(r_{1}, r_{2}\right)-\frac{1}{\pi} \log \frac{r_{2}}{r_{1}} \geq \frac{1}{\pi} \int_{r_{1}}^{r_{2}} \frac{d r}{r+C_{4} \sqrt{r}}-\frac{1}{\pi} \int_{r_{1}}^{r_{2}} \frac{d r}{r}=-\frac{1}{\pi} \int_{r_{1}}^{r_{2}} \frac{C_{4} d r}{\left(r+C_{4} \sqrt{r}\right) \sqrt{r}}$.

Since the right hand side of (18) tends to 0 as $r_{1}, r_{2} \rightarrow \infty$, (16) and (17) imply (11) and the lemma is proved.

From (10) follows that for every sufficiently large $R>0$ the preimage $\varphi^{-1}(L(r))$ for $r=\frac{2}{A} R$ and an interval of the real axis are the boundary of a domain containing $\mathbf{C}_{-} \cap\{|z|=R\}$ in its interior. Thus $\mathbf{C}_{-} \cap\{|z|=R\}$ is mapped by $\varphi$ into the component of $\Omega(s) \backslash L(r)$ not containing $r+1$. Consequently, for every $z \in \mathbf{C}_{-}$with $|z|=R$ either

or (by (12) and (13))

$$
\operatorname{Re} \varphi(z) \leq|\varphi(z)| \leq r=\frac{2}{A} R=\frac{2}{A}|z|
$$

$$
\operatorname{Re} \varphi(z) \leq C_{2}-\log \pi+\log r=C_{5}+\log R=C_{5}+\log |z|,
$$

and we have proved

$$
|F(z)|=O\left(\mathrm{e}^{C_{6}|z|}\right), \quad C_{6}:=\max \left(\frac{2}{A}, 1\right),
$$

i.e. $F(z)$ is of exponential type in $\mathbf{C}_{-}$. Since $F$ is continuous in $\overline{\mathbf{C}}_{-}$and real-valued on the real axis, it can be extended to $\mathbf{C}$ by the reflection principle and is of exponential type there.

Note that for $a>0$ also $\varphi(a z)$ is a conformal map of $\mathbf{C}_{-}$onto $\Omega(s)$. Choosing $a>0$ appropriately we can always achieve that the exponential type of $F$ is equal 
to $\pi$, and we assume this normalization for the rest of the proof for $a=1$. Then we have to show that $\left\{\lambda_{n}\right\}_{n \in \mathbf{Z}}=F^{-1}(0)$ is a complete interpolating sequence.

Lemma 2. The sequence $\left\{\lambda_{n}\right\}_{n \in \mathbf{Z}}$ is separated.

Proof. For $\zeta \in \Omega(s)$ let $L_{\zeta}(r)$ be the connected arc of $\{|\zeta-z|=r\} \cap \Omega(s)$ that contains $\zeta+r$ and $\Omega_{\zeta}(r)$ the component of $\Omega(s) \backslash L_{\zeta}(r)$ that contains $\zeta+r / 2$.

Denote by $P_{t}, t \in \mathbf{R}$ the polygonal line

$$
P_{t}:=\bigcup_{n \in \mathbf{Z}}\left\{x\left(\log \left|c_{n}\right|+\mathrm{i} n \pi\right)+(1-x)\left(\log \left|c_{n+1}\right|+\mathrm{i}(n+1) \pi\right)+t: x \in[0,1]\right\} .
$$

$P_{0}$ just connects the endpoints of the slits of $\Omega(s)$. For fixed $t>0$ and every $\zeta \in P_{t}$ the length $l_{\zeta}(r)$ of $L_{\zeta}(r)$ satisfies the estimate (14) with a constant $C_{4}>0$ that does not depend on $\zeta$ (but, of course, on $t$ ). Hence we conclude as in Lemma 1

$$
A\left(1+g_{1}(\zeta, r)\right) r \leq \inf _{z \in L_{\zeta}(r)}\left|\varphi^{-1}(z)\right| \leq \sup _{z \in L_{\zeta}(r)}\left|\varphi^{-1}(z)\right| \leq A\left(1+g_{2}(\zeta, r)\right) r, \quad r \geq r_{0}(\zeta),
$$

where $g_{1}, g_{2}$ are such that

$$
\lim _{r \rightarrow \infty} g_{j}(\zeta, r)=0, \quad j=1,2, \zeta \in P_{t} .
$$

According to [8, IV.6], [33], the harmonic measure $\omega_{\zeta}(r)$ of the boundary arc $L_{\zeta}(r)$ at the point $\zeta$ with respect to the domain $\Omega_{\zeta}(r)$ allows the estimate

$$
\omega_{\zeta}(r) \leq C_{7} \exp \left(-\pi \int_{r_{\zeta}}^{r} \frac{d \varrho}{l_{\zeta}(\varrho)}\right) \leq C_{7} \exp \left(-\int_{r_{\zeta}}^{r} \frac{d \varrho}{\varrho+C_{4} \sqrt{\varrho}}\right) \leq \frac{C_{8}}{r},
$$

where $r_{\zeta}=\operatorname{dist}(\zeta, \partial \Omega(s))>0$. For the harmonic function $-\operatorname{Im} \varphi^{-1}$ we find now

$$
-\operatorname{Im} \varphi^{-1}(\zeta) \leq \omega_{\zeta}(r) \sup _{z \in L_{\zeta}(r)}\left|\varphi^{-1}(z)\right| \leq \frac{C_{8}}{r} A\left(1+g_{2}(\zeta, r)\right) r .
$$

Letting $r \rightarrow \infty$ we conclude

$$
0>\operatorname{Im} \varphi^{-1}(\zeta) \geq-A C_{8}, \quad \zeta \in P_{t},
$$

i.e. the "left" component of $\Omega(s) \backslash P_{t}$ is mapped into some strip below the real axis.

Let $x_{n}:=\varphi^{-1}\left(\log \left|c_{n}\right|+\mathrm{i} n \pi\right)$ be the critical points of $F$ and consider the nonEuclidean segments $H_{n, m} \subset \overline{\mathbf{C}}_{-}$connecting $x_{n}$ and $x_{m}$, i.e. $H_{n, m}$ are Euclidean semicircles with centers $\left(x_{n}+x_{m}\right) / 2 . \varphi\left(H_{n, m}\right)$ is a curve connecting the endpoints of the $n$-th and $m$-th slit of $\Omega(s)$, and according to the Gehring-Hayman Theorem (see [28], Theorem 4.20) it is not much longer than any curve $\gamma_{n, m} \subset \Omega(s)$ connecting the same endpoints, i.e.

$$
\text { length }\left(\varphi\left(H_{n, m}\right)\right) \leq C_{9} \text { length }\left(\gamma_{n, m}\right),
$$

where $C_{9}>0$ is a universal constant. Choosing $\gamma_{n, n+1}$ as the straight line segment between two successive slit endpoints and noting that by (8) this segment is not longer than $\sqrt{\pi^{2}+C_{1}^{2}}$, we find that

$$
\operatorname{length}\left(\varphi\left(H_{n, n+1}\right)\right) \leq C_{9} \sqrt{\pi^{2}+C_{1}^{2}}, \quad n \in \mathbf{Z},
$$


and thus $\varphi\left(H_{n, n+1}\right) \cap P_{t}=\emptyset$ for all $n \in \mathbf{Z}$ provided that $t>0$ has been chosen sufficiently large. From (19) follows that

$$
H_{n, n+1} \subset\left\{z \in \mathbf{C}:-A C_{8} \leq \operatorname{Im} z \leq 0\right\}, \quad n \in \mathbf{Z},
$$

and hence the (Euclidean) radii of the semicircles $H_{n, n+1}$ are bounded. We infer

$$
\Delta_{1}:=\sup _{n \in \mathbf{Z}}\left|x_{n+1}-x_{n}\right|<\infty .
$$

Let $G$ be the auxiliary domain

$$
G:=\left\{x+\mathrm{i} y \in \mathbf{C}: x>\max \left(\log |y|,-C_{10}\right)\right\},
$$

where $C_{10}>0$ is chosen so large that $G$ has the property that for every $n \in \mathbf{Z}$ there is $\zeta_{n} \in \mathbf{C}$ such that $G+\zeta_{n} \subset \Omega(s)$ and $-C_{10}+1+\zeta_{n}$ is "left" of $P_{t_{0}}$ for $t_{0}=-C_{9} \sqrt{\pi^{2}+C_{1}^{2}}$. Let further $\psi: \mathbf{C}_{-} \rightarrow G$ be a conformal mapping with

$$
\psi(\infty)=\infty, \quad \lim _{|z| \rightarrow \infty} \frac{|\psi(z)|}{|z|}=A,
$$

and let $y_{0}<0$ be so close to 0 that $-C_{10}+1 \in \psi\left(\mathbf{R}+\mathrm{i} y_{0}\right)$. Then $\zeta_{n}+\psi(z)$ maps $\mathbf{C}_{-}$ onto $G+\zeta_{n}$, and according to the Lindelöf principle (cf. [16, IV.§1.61]) $\varphi\left(\mathbf{R}+\mathrm{i} y_{0}\right)$ lies in the "left" component of $\Omega(s) \backslash\left(\psi\left(\mathbf{R}+\mathrm{i} y_{0}\right)+\zeta_{n}\right)$, see Figure 2 .

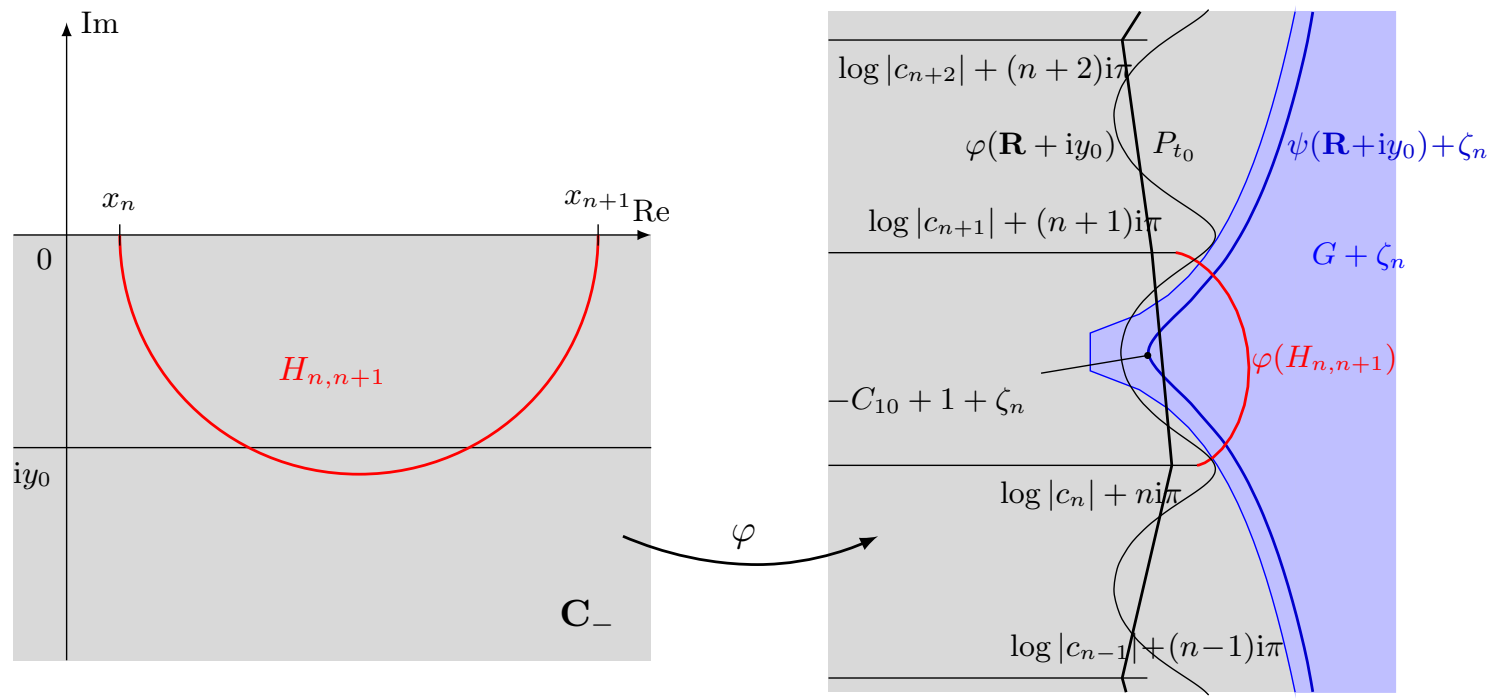

Figure 2. The auxiliary domain $G+\zeta_{n}$.

Since $\varphi\left(H_{n, n+1}\right)$ lies to the right of $P_{t_{0}}$, the curves $\varphi\left(\mathbf{R}+\mathrm{i} y_{0}\right)$ and $\varphi\left(H_{n, n+1}\right)$ have a non-empty intersection. Hence also $\left(\mathbf{R}+\mathrm{i} y_{0}\right) \cap H_{n, n+1} \neq \emptyset$ for all $n \in \mathbf{Z}$ and thus the (Euclidean) radius of $H_{n, n+1}$ cannot be smaller than $\left|y_{0}\right|$. We infer

$$
\delta_{1}:=\inf _{n \in \mathbf{Z}}\left|x_{n}-x_{n+1}\right|>0 .
$$

Let the zero set $\left\{\lambda_{n}\right\}_{n \in \mathbf{Z}}=F^{-1}(0)=\varphi^{-1}(-\infty)$ be numbered such that $x_{n}<\lambda_{n}<$ $x_{n+1}$ for all $n \in \mathbf{Z}$. Let $E_{n}$ be the set $E_{n}:=\left\{x+\mathrm{i} n \pi: x \leq \log \left|c_{n}\right|-2 C_{1}\right\} \cup\{x+$ $\left.\mathrm{i}(n+1) \pi: x \leq \log \left|x_{n+1}\right|-2 C_{1}\right\}$ and let $\tilde{E}_{n} \subset\left(x_{n}, x_{n+1}\right)$ be such that $\varphi\left(\tilde{E}_{n}\right)=E_{n}$. 
We look at the family $\Gamma_{1}^{(n)}$ of curves connecting $\tilde{E}_{n}$ with $\left[x_{n+1}, \infty\right)$ in $\mathbf{C}_{-}$and at the family $\Gamma_{2}^{(n)}$ of curves connecting $\tilde{E}_{n}$ with $\left(-\infty, x_{n}\right]$ in $\mathbf{C}_{-}$. Further let

$$
\begin{aligned}
& E_{n}^{ \pm}:=\bigcup_{k=n+1}^{\infty}\left\{x+\mathrm{i} k \pi: x \leq \log \left|c_{n}\right| \pm C_{1} \pm \log |k-n|\right\}, \\
& \Omega_{n}^{ \pm}:=\mathbf{C} \backslash \bigcup_{k \in \mathbf{Z} \backslash\{n\}}\left\{x+\mathrm{i} k \pi: x \leq \log \left|c_{n}\right| \mp C_{1} \mp \log |k-n|\right\} \cup\left\{x+\mathrm{i} n \pi: x \leq \log \left|c_{n}\right|\right\},
\end{aligned}
$$

and $\Gamma_{-}^{(n)}$ the family of curves $\gamma$ connecting $E_{n}$ with $E_{n}^{-}$in $\Omega_{n}^{-}$such that if $\gamma:(0,1) \rightarrow$ C then we have $n \pi<\operatorname{Im} \gamma(t)<(n+1) \pi$ for $t$ sufficiently close to 0 and $\operatorname{Im} \gamma(t)>(n+$ 1) $\pi$ for $t$ sufficiently close to 1 . Similarly, let $\Gamma_{+}^{(n)}$ be the family of curves $\gamma$ connecting $E_{n}$ with $E_{n}^{+}$in $\Omega_{n}^{+}$such that if $\gamma:(0,1) \rightarrow \mathbf{C}$ then we have $n \pi<\operatorname{Im} \gamma(t)<(n+1) \pi$ for $t$ sufficiently close to 0 and $\operatorname{Im} \gamma(t)>(n+1) \pi$ for $t$ sufficiently close to 1 . We have

$$
\bmod \Gamma_{-}^{(n)} \leq \bmod \varphi\left(\Gamma_{1}^{(n)}\right) \leq \bmod \Gamma_{+}^{(n)}
$$

by the monotonicity properties of the module. Since all domains $\Omega_{n}^{+}\left(\Omega_{n}^{-}\right)$and all sets $E_{n}^{+}\left(E_{n}^{-}\right)$differ only by a translation, the upper (lower) bound in (23) is in fact independent of $n \in \mathbf{Z}$. By the conformal invariance of the module we have thus

$$
0<C_{11} \leq \bmod \Gamma_{1}^{(n)} \leq C_{12}<\infty
$$

and a similar estimate holds for $\Gamma_{2}^{(n)}$. From the subsequent Lemma 8 we obtain the existence of $\delta_{2} \in(0,1)$ such that

$$
\tilde{E}_{n} \subset\left[\frac{x_{n}+x_{n+1}}{2}-\delta_{2} \frac{x_{n+1}-x_{n}}{2}, \frac{x_{n}+x_{n+1}}{2}+\delta_{2} \frac{x_{n+1}-x_{n}}{2}\right], \quad n \in \mathbf{Z} .
$$

Since $\lambda_{n} \in \tilde{E}_{n},(22) \operatorname{implies}_{\inf _{n \in \mathbf{Z}}}\left|x_{n}-\lambda_{n}\right|>0, \inf _{n \in \mathbf{Z}}\left|x_{n+1}-\lambda_{n}\right|>0$, and $\delta:=$ $\inf _{n \in \mathbf{Z}}\left|\lambda_{n}-\lambda_{n+1}\right|>0$. Hence the set $\left\{\lambda_{n}\right\}_{n \in \mathbf{Z}}$ is separated and condition (i) of Theorem 2 is fulfilled.

Condition (ii) of Theorem 2 is now quite easy to establish, since the limit

$$
F(z)=F(0) \lim _{R \rightarrow \infty} \prod_{\left|\lambda_{n}\right|<R}\left(1-\frac{z}{\lambda_{n}}\right)
$$

is known to exist for all functions $F$ of the Cartwright class, i.e. entire functions $F$ of exponential type such that

$$
\int_{-\infty}^{\infty} \frac{\log ^{+}|F(t)|}{1+t^{2}} d t<\infty
$$

see [19], p. 130.

Lemma 3. F belongs to the Cartwright class.

Proof. We have to show the existence of the integral (24). Relation (3) implies

$$
\left|\lambda_{n}\right| \geq-\left|\lambda_{0}\right|+|n| \delta, \quad n \in \mathbf{Z},
$$


and (21) implies that also (5) holds. For $t \in\left(\lambda_{n-1}, \lambda_{n}\right)$ we know that $\varphi(t)$ is on a slit of $\Omega(s)$ such that $\operatorname{Im} \varphi(t)=n \pi$ and $\operatorname{Re} \varphi(t) \leq \log \left|c_{n}\right|$. Hence by $(9)$

$$
\log |F(t)|=\operatorname{Re} \varphi(t) \leq C_{2}+\log |n|,
$$

and also

$$
\log ^{+}|F(t)| \leq C_{2}+\log |n|
$$

for all $|n| \geq n_{0}$ such that the right hand side of this inequality is positive. We obtain

$$
\begin{aligned}
\int_{\lambda_{n-1}}^{\lambda_{n}} \frac{\log ^{+}|F(t)|}{1+t^{2}} d t & \leq\left(C_{2}+\log |n|\right) \int_{\lambda_{n-1}}^{\lambda_{n}} \frac{d t}{1+t^{2}} \leq \frac{\left(C_{2}+\log |n|\right)\left(\lambda_{n}-\lambda_{n-1}\right)}{1+\min \left(\left|\lambda_{n-1}\right|,\left|\lambda_{n}\right|\right)^{2}} \\
& \leq \frac{\left(C_{2}+\log |n|\right) \Delta}{1+\left(-\left|\lambda_{0}\right|+(|n|-1) \delta\right)^{2}} \leq C_{13} \frac{\log |n|}{|n|^{2}}
\end{aligned}
$$

provided that $|n| \geq n_{1}$, where $n_{1} \geq n_{0}$ is such that $-\left|\lambda_{0}\right|+\left(n_{1}-1\right) \delta>0$. Consequently,

$$
\int_{-\infty}^{\infty} \frac{\log ^{+}|F(t)|}{1+t^{2}} d t \leq \int_{\lambda_{-n_{1}}}^{\lambda_{n_{1}+1}} \frac{\log ^{+}|F(t)|}{1+t^{2}} d t+C_{13} \sum_{|n| \geq n_{1}} \frac{\log |n|}{|n|^{2}}<\infty .
$$

Next we have to show condition (iii) of Theorem 2, i.e. the Muckenhoupt condition $\left(A_{2}\right)$. For that purpose we choose $y<0$ such that the line $\mathrm{i} y+\mathbf{R}$ is disjoint from all semicircles $H_{n, n+1}(n \in \mathbf{Z})$, which is possible in view of (21). Let $I_{n}:=\left[\left(x_{n-1}+x_{n}\right) / 2\left(x_{n}+x_{n+1}\right) / 2\right]$. According to (3) for some integer $N>0$ the intervals $I_{n}+\mathrm{i} y$ lie inside $H_{n-N, n+N}$ for all $n \in \mathbf{Z}$. Using again the Gehring-Hayman Theorem we find that there is $C_{14}>0$ with

$$
\left|\operatorname{Re}\left(\varphi\left(x_{n}\right)-\varphi(z)\right)\right|<C_{14}, \quad z \in I_{n}+\mathrm{i} y, n \in \mathbf{Z} .
$$

This implies immediately

$$
\left|\mathrm{e}^{\varphi(z)}\right| \leq\left|c_{n}\right| \mathrm{e}^{C_{14}} \quad \text { and } \quad\left|\mathrm{e}^{-\varphi(z)}\right| \leq\left|c_{n}\right|^{-1} \mathrm{e}^{C_{14}}
$$

for $z \in I_{n}+\mathrm{i} y, n \in \mathbf{Z}$.

Given any interval $I \subset \mathbf{R}$ we denote by $I_{p}, \ldots, I_{q}$ all intervals among $\left\{I_{n}\right\}_{n \in \mathbf{Z}}$ that are not disjoint with $I$. Further we find

$$
\int_{I_{n}}|F(x+\mathrm{i} y)|^{2} d x \leq c_{n}^{2} \mathrm{e}^{2 C_{14}} \Delta_{1}, \quad \int_{I_{n}}|F(x+\mathrm{i} y)|^{-2} d x \leq c_{n}^{-2} \mathrm{e}^{2 C_{14}} \Delta_{1}, \quad n=p, \ldots, q .
$$

Adding these inequalities for $n=p, \ldots, q$ and multiplying the results yields

$$
\begin{aligned}
\int_{I}|F(x+\mathrm{i} y)|^{2} d x \int_{I}|F(x+\mathrm{i} y)|^{-2} d x & \leq \sum_{n=p}^{q} \int_{I_{n}}|F(x+\mathrm{i} y)|^{2} d x \sum_{n=p}^{q} \int_{I_{n}}|F(x+\mathrm{i} y)|^{-2} d x \\
& \leq \mathrm{e}^{4 C_{14}} \Delta_{1}^{2} \sum_{n=p}^{q} c_{n}^{2} \sum_{n=p}^{q} c_{n}^{-2} .
\end{aligned}
$$

First assume $q-p>1$. Since at least $(q-p-1)$ of the intervals $I_{p}, \ldots, I_{q}$ are contained in $I$ we have $|I| \geq \delta_{1}(q-p-1) \geq \frac{\delta_{1}}{3}(q-p+1)$, and using the assumption 
$\left(\tilde{A}_{2}\right)$ we get

$$
\int_{I}|F(x+\mathrm{i} y)|^{2} d x \int_{I}|F(x+\mathrm{i} y)|^{-2} d x \leq \mathrm{e}^{4 C_{14}} \Delta_{1}^{2} C \frac{9}{\delta_{1}^{2}}|I|^{2},
$$

and thus $\left(A_{2}\right)$.

In the special case $p=q$ the interval $I$ is contained in $I_{p}=I_{q}$ and we can estimate

$$
\int_{I}|F(x+\mathrm{i} y)|^{2} d x \int_{I}|F(x+\mathrm{i} y)|^{-2} d x \leq c_{p}^{2} \mathrm{e}^{2 C_{14}}|I| c_{p}^{-2} \mathrm{e}^{2 C_{14}}|I|=\mathrm{e}^{4 C_{14}}|I|^{2} .
$$

If finally $q=p+1$ then the interval $I$ is contained in $I_{p} \cup I_{p+1}$. Using $c_{p}^{2} /(4 C) \leq$ $c_{p+1}^{2} \leq 4 C c_{p}^{2}$ we handle this case in the following way:

$$
\begin{aligned}
\int_{I}|F(x+\mathrm{i} y)|^{2} d x \int_{I}|F(x+\mathrm{i} y)|^{-2} d x & \leq\left(c_{p}^{2}+c_{p+1}^{2}\right) \mathrm{e}^{2 C_{14}}|I|\left(c_{p}^{-2}+c_{p+1}^{-2}\right) \mathrm{e}^{2 C_{14}}|I| \\
& \leq(1+4 C)^{2} \mathrm{e}^{4 C_{14}}|I|^{2} .
\end{aligned}
$$

The Muckenhoupt condition is hence always fulfilled and Theorem 2 implies that $\left\{\lambda_{n}\right\}_{n \in \mathbf{Z}}$ is a complete interpolating sequence.

Next we have to show that every complete interpolating sequence $\left\{\lambda_{n}\right\}_{n \in \mathbf{Z}}$ can be obtained in this way. From (ii) of Theorem 2 we know that the numbers $\left\{\lambda_{n}\right\}_{n \in \mathbf{Z}}$ are the zeros of an entire function $F$ of exponential type $\pi$, which also belongs to the Laguerre-Pólya class $\mathscr{L} \mathscr{P}$. We can assume that $(-1)^{n} c_{n} \geq 0$ holds for the sequence $\left\{c_{n}\right\}_{n \in \mathbf{Z}}=\operatorname{Cr} F$, otherwise we consider $-F$. Hence we have the representation (6) with a conformal map $\varphi: \mathbf{C}_{-} \rightarrow \Omega(\mathrm{Cr} F)$. Letting $R \rightarrow \infty$ in

$$
\prod_{\left|\lambda_{n}\right|<R}\left|1-\frac{\mathrm{i} y}{\lambda_{n}}\right|^{2}=\prod_{\left|\lambda_{n}\right|<R}\left(1+\frac{y^{2}}{\lambda_{n}^{2}}\right) \geq \sum_{\left|\lambda_{n}\right|<R} \frac{y^{2}}{\lambda_{n}^{2}}, \quad y \in \mathbf{R},
$$

we obtain

$$
|F(\mathrm{i} y)| \geq|y|\left(\sum_{n \in \mathbf{Z}} \frac{1}{\lambda_{n}^{2}}\right)^{1 / 2}, \quad y \in \mathbf{R},
$$

and hence $\lim _{y \rightarrow-\infty} \operatorname{Re} \varphi(\mathrm{i} y)=\lim _{y \rightarrow-\infty} \log |F(\mathrm{i} y)|=\infty$.

It remains to show condition $\left(\tilde{A}_{2}\right)$ for the sequence $\left\{d_{n}\right\}_{n \in \mathbf{Z}}=\left\{c_{n}^{2}\right\}_{n \in \mathbf{Z}}$. Fix $y<0$ and recall that $w(x)=|F(x+\mathrm{i} y)|^{2}$ satisfies the Muckenhoupt condition $\left(A_{2}\right)$ with a constant $C>0$. Next we need that the distance between neighboring slit endpoints is bounded.

Lemma 4. There is $C_{15}>0$ such that

$$
|\log | c_{n}|-\log | c_{n+1}|| \leq C_{15} \quad \forall n \in \mathbf{Z} .
$$

Proof. By Lemma 6.5 of chapter VI of [7], the function $\psi(x):=\frac{1}{2} \log w(x)=$ $\log |F(x+\mathrm{i} y)|=\operatorname{Re} \varphi(x+\mathrm{i} y)$ belongs to the space $\operatorname{BMO}(\mathbf{R})$, i.e.

$$
\|\psi\|_{*}:=\sup _{I} \frac{1}{|I|} \int_{I}\left|\psi(x)-\psi_{I}\right| d x<\infty, \quad \psi_{I}:=\frac{1}{|I|} \int_{I} \psi(x) d x,
$$

where the supremum is taken over all bounded intervals. This implies (Ibid., Theorem 1.2) that

$$
\int_{\mathbf{R}} \frac{\log ^{+}|F(x+\mathrm{i} y)|}{1+x^{2}} d x \leq \int_{\mathbf{R}} \frac{|\psi(x)|}{1+x^{2}} d x<\infty
$$


and the representation

$$
\begin{aligned}
\log |F(x)|= & B|y|+\sum_{n \in \mathbf{Z}} \log \left|\frac{1-(x-\mathrm{i} y) /\left(\lambda_{n}-\mathrm{i} y\right)}{1-(x-\mathrm{i} y) /\left(\lambda_{n}+\mathrm{i} y\right)}\right| \\
& +\frac{1}{\pi} \int_{\mathbf{R}} \frac{|y|}{|x-\mathrm{i} y-t|^{2}} \log \mid F(t+\mathrm{i} y) d t, \quad x \in \mathbf{R},
\end{aligned}
$$

with $B:=\lim \sup _{t \rightarrow \infty} \log |F(\mathrm{it})| / t$ is valid, cf. section III.G.3 of [14]. This formula shows among other things that $\operatorname{Re} \varphi$ restricted to $\mathbf{R}$ has logarithmic singularities in the points $\lambda_{n}$, whereas on $\mathbf{R}+\mathrm{i} y$ this function is continuous. Therefore we cannot compare $\operatorname{Re} \varphi$ on $\mathbf{R}$ and $\mathbf{R}+\mathrm{i} y$ directly, but have to resort to mean values. Let $\delta>0$ be the separation constant defined in $(3)$ and let $I_{x}(x \in \mathbf{R})$ be the interval of length $\delta$ centered at $x$. By Lemma 1.1(b) of chapter VI of $[7]^{2}$ we know that

$$
\left|\psi_{I_{x_{1}}}-\psi_{I_{x_{2}}}\right| \leq C_{16} \log \left(1+\frac{1}{\delta}\left|x_{1}-x_{2}\right|\right)
$$

for all $x_{1}, x_{2} \in \mathbf{R}$ and some constant $C_{16}>0$. Let $x \in \mathbf{R} \backslash\left\{\lambda_{n}\right\}_{n \in \mathbf{Z}}$ and assume $\lambda_{n-1}<x<\lambda_{n}$ for some $n \in \mathbf{Z}$. Then $\left|x-\lambda_{k}\right| \geq \delta(k-n)$ for $k \geq n$ and $\left|x-\lambda_{k}\right| \geq$ $\delta(n-k-1)$ for $k \leq n-1$, so that we find

$$
\begin{aligned}
0 & >\sum_{k \in \mathbf{Z} \backslash\{n-1, n\}} \log \left|\frac{1-(x-\mathrm{i} y) /\left(\lambda_{k}-\mathrm{i} y\right)}{1-(x-\mathrm{i} y) /\left(\lambda_{k}+\mathrm{i} y\right)}\right| \\
& =\sum_{k \in \mathbf{Z} \backslash\{n-1, n\}} \frac{1}{2} \log \left(1-\frac{4 y^{2}}{\left(\lambda_{k}-x\right)^{2}+4 y^{2}}\right) \\
& \geq \sum_{k=1}^{\infty} \log \left(1-\frac{4 y^{2}}{k^{2} \delta^{2}+4 y^{2}}\right)=: C_{17}>-\infty
\end{aligned}
$$

If $I \subset\left[\lambda_{n-1}, \lambda_{n}\right]$ is an interval we obtain

$$
\begin{aligned}
0 & >\int_{I} \log \left|\frac{1-(x-\mathrm{i} y) /\left(\lambda_{n-1}-\mathrm{i} y\right)}{1-(x-\mathrm{i} y) /\left(\lambda_{n-1}+\mathrm{i} y\right)}\right|+\log \left|\frac{1-(x-\mathrm{i} y) /\left(\lambda_{n}-\mathrm{i} y\right)}{1-(x-\mathrm{i} y) /\left(\lambda_{n}+\mathrm{i} y\right)}\right| d x \\
& \geq \int_{\lambda_{n-1}}^{\lambda_{n}} \log \left(1-\frac{4 y^{2}}{\left(\lambda_{n-1}-x\right)^{2}+4 y^{2}}\right)+\log \left(1-\frac{4 y^{2}}{\left(\lambda_{n}-x\right)^{2}+4 y^{2}}\right) d x \\
& =4 y \arctan \frac{\lambda_{n-1}-\lambda_{n}}{2 y}-2\left(\lambda_{n-1}-\lambda_{n}\right) \log \left(1-\frac{4 y^{2}}{\left(\lambda_{n-1}-\lambda_{n}\right)^{2}+4 y^{2}}\right) \\
& \geq C_{18}>-\infty
\end{aligned}
$$

where $C_{18}$ is independent of $I$ and $n$ since the function

$$
\lambda \mapsto 4 y \arctan \frac{\lambda}{2 y}-2 \lambda \log \left(1-\frac{4 y^{2}}{\lambda^{2}+4 y^{2}}\right)
$$

\footnotetext{
${ }^{2}$ Inequality (8) can be interpreted as a discrete analogue of this lemma. That it holds is particularly delicate in view of an example of Avdonin [2] who constructed a complete interpolating sequence so that $\psi$ oscillates so much that $\sup _{\left|x_{1}-x_{2}\right| \leq 1}\left|\psi\left(x_{1}\right)-\psi\left(x_{2}\right)\right|=\infty$.
} 
is bounded on $\mathbf{R}$. Altogether we have shown that for intervals $I$ not containing any $\lambda_{n}$ in its interior

$$
0>\frac{1}{|I|} \int_{I} \sum_{n \in \mathbf{Z}} \log \left|\frac{1-(x-\mathrm{i} y) /\left(\lambda_{n}-\mathrm{i} y\right)}{1-(x-\mathrm{i} y) /\left(\lambda_{n}+\mathrm{i} y\right)}\right| d x \geq C_{17}+\frac{1}{|I|} C_{18} .
$$

For any interval $I_{\tilde{x}}$ we have

$$
\begin{aligned}
& \frac{1}{\left|I_{\tilde{x}}\right|} \int_{I_{\tilde{x}}} \frac{1}{\pi} \int_{\mathbf{R}} \frac{|y|}{|x-\mathrm{i} y-t|^{2}} \log |F(t+\mathrm{i} y)| d t d x \\
& =\frac{1}{\delta} \int_{I_{\tilde{x}}} \frac{1}{\pi} \int_{\mathbf{R}} \frac{|y|}{|\tilde{x}-\mathrm{i} y-s|^{2}} \log |F(s+x-\tilde{x}+\mathrm{i} y)| d s d x \\
& =\frac{1}{\pi} \int_{\mathbf{R}} \frac{|y|}{|\tilde{x}-\mathrm{i} y-s|^{2}} \frac{1}{\delta} \int_{I_{\tilde{x}}} \log |F(s+x-\tilde{x}+\mathrm{i} y)| d x d s \\
& =\frac{1}{\pi} \int_{\mathbf{R}} \frac{|y|}{|\tilde{x}-\mathrm{i} y-s|^{2}} \psi_{I_{s}} d s
\end{aligned}
$$

and the latter integral can be estimated using (27) in the following way

$$
\begin{aligned}
& \left|\frac{1}{\pi} \int_{\mathbf{R}} \frac{|y|}{|\tilde{x}-\mathrm{i} y-s|^{2}} \psi_{I_{s}} d s-\psi_{I_{\tilde{x}}}\right| \\
& =\left|\frac{1}{\pi} \int_{\mathbf{R}} \frac{|y|}{|\tilde{x}-\mathrm{i} y-s|^{2}} \psi_{I_{s}} d s-\frac{1}{\pi} \int_{\mathbf{R}} \frac{|y|}{|\tilde{x}-\mathrm{i} y-s|^{2}} \psi_{I_{\tilde{x}}} d s\right| \\
& \leq \frac{1}{\pi} \int_{\mathbf{R}} \frac{|y| C_{16} \log \left(1+\frac{1}{\delta}|\tilde{x}-s|\right)}{|\tilde{x}-\mathrm{i} y-s|^{2}} d s=\frac{C_{16}}{\pi} \int_{\mathbf{R}} \frac{|y| \log (1+|t| / \delta)}{t^{2}+y^{2}} d t=: C_{19}<\infty .
\end{aligned}
$$

Combining this with (26), (28), and (29) we arrive at

$$
\left|\frac{1}{\left|I_{\tilde{x}}\right|} \int_{I_{\tilde{x}}} \log \right| F(x)\left|d x-\psi_{I_{\tilde{x}}}\right| \leq B|y|+\left|C_{17}\right|+\frac{1}{\delta}\left|C_{18}\right|+C_{19}=: C_{20}
$$

for all intervals $I_{\tilde{x}}$ of length $\delta$ centered at $\tilde{x}$ and not containing any $\lambda_{n}$ in its interior. Assume that $\tilde{x}_{n}$ is such that $I_{\tilde{x}_{n}} \subset\left[\lambda_{n-1}, \lambda_{n}\right]$. Then we have $\operatorname{Re} \varphi(x) \leq \log \left|c_{n}\right|$ for all $x \in I_{\tilde{x}_{n}}$ and thus

$$
\frac{1}{\left|I_{\tilde{x}_{n}}\right|} \int_{I_{\tilde{x}_{n}}} \log |F(x)| d x \leq \log \left|c_{n}\right|, \quad n \in \mathbf{Z}
$$

and also

$$
\psi_{\tilde{x}_{n}}-C_{20} \leq \log \left|c_{n}\right|, \quad n \in \mathbf{Z} .
$$

In order to get an upper bound for $\log \left|c_{n}\right|$ we recall that $\operatorname{Re} \varphi\left(x_{n}\right)=\log \left|c_{n}\right|$ for some $x_{n} \in\left(\lambda_{n-1}, \lambda_{n}\right)$ and hence (26) yields

$$
\log \left|c_{n}\right| \leq B|y|+\frac{1}{\pi} \int_{\mathbf{R}} \frac{|y|}{\left|x_{n}-\mathrm{i} y-t\right|^{2}} \log |F(t+\mathrm{i} y)| d t
$$


Now we compute

$$
\begin{aligned}
& \left|\frac{1}{\pi} \int_{\mathbf{R}} \frac{|y|}{\left|x_{n}-\mathrm{i} y-t\right|^{2}} \log \right| F(t+\mathrm{i} y)\left|d t-\psi_{I_{\tilde{x}_{n}}}\right| \\
& =\left|\frac{1}{\pi} \int_{\mathbf{R}} \frac{|y|}{\left|x_{n}-\mathrm{i} y-t\right|^{2}}\left(\psi(t)-\psi_{I_{\tilde{x}_{n}}}\right) d t\right| \\
& \leq \frac{1}{\pi} \sum_{k \in \mathbf{Z}_{I_{\tilde{x}_{n}}+k \delta}} \int_{\frac{|y|}{\left(x_{n}-t\right)^{2}+y^{2}}}\left(\left|\psi(t)-\psi_{I_{\tilde{x}_{n}}+k \delta}\right|+\left|\psi_{I_{\tilde{x}_{n}}+k \delta}-\psi_{I_{\tilde{x}_{n}}}\right|\right) d t .
\end{aligned}
$$

For $t \in I_{\tilde{x}_{n}}+k \delta$ and $k \geq 0$ we have $t-x_{n} \geq\left(\lambda_{n-1}+k \delta\right)-\lambda_{n} \geq k \delta-\Delta$, where $\Delta$ is defined in (5). For $k \leq 0$ we find similarly $x_{n}-t \geq-k \delta-\Delta$. Thus for all $k$ with $|k|>k_{0}:=\Delta / \delta$ we have $\left|x_{n}-t\right| \geq|k| \delta-\Delta>0$ and we can continue the previous chain of inequalities using (27)

$$
\begin{aligned}
\ldots \leq & \frac{1}{\pi} \sum_{|k| \leq k_{0}} \frac{\delta}{|y|}\left(\frac{1}{\delta} \int_{I_{\tilde{x}_{n}}+k \delta}\left|\psi(t)-\psi_{\tilde{x}_{n}+k \delta}\right| d t+C_{16} \log (1+|k|)\right) \\
& +\frac{1}{\pi} \sum_{|k|>k_{0}} \frac{|y| \delta}{(|k| \delta-\Delta)^{2}+y^{2}}\left(\frac{1}{\delta} \int_{I_{\tilde{x}_{n}}+k \delta}\left|\psi(t)-\psi_{I_{\tilde{x}_{n}}+k \delta}\right| d t+C_{16} \log (1+|k|)\right) \\
\leq & \frac{\|\psi\|_{*}}{\pi}\left(\sum_{|k| \leq k_{0}} \frac{\delta}{|y|}+\sum_{|k|>k_{0}} \frac{|y| \delta}{(|k| \delta-\Delta)^{2}+y^{2}}\right) \\
& +\frac{C_{16}}{\pi}\left(\sum_{|k| \leq k_{0}} \frac{\delta}{|y|} \log (1+|k|)+\sum_{|k|>k_{0}} \frac{|y| \delta \log (1+|k|)}{(|k| \delta-\Delta)^{2}+y^{2}}\right)=: C_{21}<\infty .
\end{aligned}
$$

In view of (31) we get thus the upper estimate

$$
\log \left|c_{n}\right| \leq B|y|+\psi_{\tilde{x}_{n}}+C_{21}, \quad n \in \mathbf{Z} .
$$

Considering also (30) we find

$$
|\log | c_{n}\left|-\psi_{\tilde{x}_{n}}\right| \leq C_{22}, \quad n \in \mathbf{Z}
$$

where $C_{22}:=\max \left(C_{20}, B|y|+C_{21}\right)$. Using once more (27) we conclude (25) with the constant $C_{15}:=2 C_{22}+C_{16} \log (1+2 \Delta / \delta)$, and the lemma is proved.

Condition (5) implies that $\sup _{n \in \mathbf{Z}}\left|x_{n+1}-x_{n}\right| \leq 2 \Delta$, and hence $\mathbf{R}+\mathrm{i} y$ is disjoint with all semicircles $H_{n, n+1}$ connecting $x_{n}$ and $x_{n+1}$ in $\mathbf{C}_{-}$, provided that $y$ had been chosen so that $|y|>2 \Delta$. Condition (3) makes it possible to choose $N>0$ so large that the intervals $\left[\lambda_{n-1}, \lambda_{n}\right]+\mathrm{i} y$ lie inside the semicircles $H_{n-N, n+N}$ for all $n \in \mathbf{Z}$. The Gehring-Hayman Theorem and (25) give the estimate

$$
\operatorname{length}\left(\varphi\left(H_{n, n+1}\right)\right) \leq C_{9} \sqrt{\pi^{2}+C_{15}^{2}}, \quad \operatorname{length}\left(\varphi\left(H_{n-N, n+N}\right)\right) \leq 2 N C_{9} \sqrt{\pi^{2}+C_{15}^{2}} .
$$

Hence

$$
\left|\operatorname{Re}\left(\varphi\left(x_{n}\right)-\varphi(z)\right)\right|<C_{23}, \quad z \in\left[\lambda_{n-1}, \lambda_{n}\right]+\mathrm{i} y, n \in \mathbf{Z},
$$

with a constant $C_{23}>0$, and also

$$
\left|c_{n}\right| \leq \mathrm{e}^{C_{23}}\left|\mathrm{e}^{\varphi(z)}\right|, \quad\left|c_{n}\right|^{-1} \leq \mathrm{e}^{C_{23}}\left|\mathrm{e}^{-\varphi(z)}\right|, \quad z \in\left[\lambda_{n-1}, \lambda_{n}\right]+\mathrm{i} y, n \in \mathbf{Z} .
$$


For any set $I=\{p, \ldots, q\}$ of consecutive integers we exploit $\left(A_{2}\right)$ for the interval $\left[\lambda_{p-1}, \lambda_{q}\right]$ to obtain

$$
\begin{aligned}
\sum_{n=p}^{q} c_{n}^{2} \sum_{n=p}^{q} c_{n}^{-2} & \leq \sum_{n=p}^{q} \frac{1}{\lambda_{n}-\lambda_{n-1}} \int_{\lambda_{n-1}}^{\lambda_{n}} \mathrm{e}^{2 C_{23}}\left|\mathrm{e}^{\varphi(x+\mathrm{i} y)}\right|^{2} d x \cdots \\
& \sum_{n=p}^{q} \frac{1}{\lambda_{n}-\lambda_{n-1}} \int_{\lambda_{n-1}}^{\lambda_{n}} \mathrm{e}^{2 C_{23}}\left|\mathrm{e}^{-\varphi(x+\mathrm{i} y)}\right|^{2} d x \\
\leq & \frac{\mathrm{e}^{4 C_{23}}}{\delta^{2}} \int_{\lambda_{p-1}}^{\lambda_{q}} w(x) d x \int_{\lambda_{p-1}}^{\lambda_{q}} \frac{1}{w(x)} d x \\
\leq & \frac{\mathrm{e}^{4 C_{23}}}{\delta^{2}} C\left(\lambda_{q}-\lambda_{p-1}\right)^{2} \leq \frac{\mathrm{e}^{4 C_{23}}}{\delta^{2}} C \Delta^{2}|I|^{2},
\end{aligned}
$$

i.e. $\left(\tilde{A}_{2}\right)$ is established and we are done.

\section{Sufficient conditions for complete interpolating sequences}

This section serves to shed some light on the result obtained in the the previous chapter. As before let $\left\{c_{n}\right\}_{n \in \mathbf{Z}}$ be the sequence of critical values of an entire function in the Laguerre-Pólya class $\mathscr{L} \mathscr{P}$. It is easy to see that condition (7) with constants $C, c>0$ implies the discrete Muckenhoupt condition $\left(\tilde{A}_{2}\right)$ for the numbers $d_{n}=c_{n}^{2}$. In view of the criterion of Eremenko and Sodin in Theorem 5, Theorem 3 is contained in Theorem 6 . This can be regarded as the discrete analogue of the observation that Theorem 2 contains Theorem 3 as a special case, since sine-type functions are easily seen to fulfill the Muckenhoupt condition $\left(A_{2}\right)$.

On the other hand, the sequence

$$
d_{n}=(1+|n|)^{2 \alpha}, \quad-\frac{1}{2}<\alpha<\frac{1}{2},
$$

is not bounded away from 0 (for $-\frac{1}{2}<\alpha<0$ ) or $\infty$ (for $0<\alpha<\frac{1}{2}$ ), but satisfies $\left(\tilde{A}_{2}\right)$. Hence the generating function of a complete interpolating sequence is not always of sine-type. That the discrete Muckenhoupt condition is indeed fulfilled for this example follows from the subsequent sufficient condition. For two positive sequences $a_{n}$ and $b_{n}$, we write $a_{n} \asymp b_{n}$ if the ratio $a_{n} / b_{n}$ is bounded from above and below by positive constants that are independent of $n$.

Lemma 5. If the condition $d_{n} \asymp(1+|n|)^{2 \alpha}$ holds for a sequence $\left\{d_{n}\right\}_{n \in \mathbf{Z}}$ and some $\alpha \in\left(-\frac{1}{2}, \frac{1}{2}\right)$, then the discrete Muckenhoupt condition $\left(\tilde{A}_{2}\right)$ is fulfilled.

Proof. The assumption $d_{n} \asymp(1+|n|)^{2 \alpha}$ means that there are constants $C_{1}, C_{2}>0$ such that

$$
C_{1}(1+|n|)^{2 \alpha} \leq d_{n} \leq C_{2}(1+|n|)^{2 \alpha}, \quad \forall n \in \mathbf{Z}
$$


For every set $I=\{p, \ldots, q\}$ of consecutive integers we can estimate

$$
\begin{aligned}
\sum_{n \in I} d_{n} \sum_{n \in I} d_{n}^{-1} & \leq C_{2} \sum_{n=p}^{q}(1+|n|)^{2 \alpha} \cdot C_{1}^{-1} \sum_{n=p}^{q}(1+|n|)^{-2 \alpha} \\
& \leq C_{2} C_{1}^{-1} \int_{p-1}^{q+1}(1+|x|)^{2 \alpha} d x \int_{p-1}^{q+1}(1+|x|)^{-2 \alpha} d x .
\end{aligned}
$$

First consider the case $0<p<q$. Using the left inequality proved in Lemma 9 below we find

$$
\begin{aligned}
\sum_{n \in I} d_{n} \sum_{n \in I} d_{n}^{-1} & \leq \frac{C_{2}}{C_{1}(1-2 \alpha)(1+2 \alpha)}\left[(2+q)^{1-2 \alpha}-p^{1-2 \alpha}\right]\left[(2+q)^{1+2 \alpha}-p^{1+2 \alpha}\right] \\
& =C_{3}\left[(2+q)^{2}-p^{1-2 \alpha}(2+q)^{1+2 \alpha}-(2+q)^{1-2 \alpha} p^{1+2 \alpha}+p^{2}\right] \\
& \leq C_{3}\left[(2+q)^{2}-2(2+q) p+p^{2}\right]=C_{3}(q-p+2)^{2} \\
& \leq 4 C_{3}(q-p+1)^{2}=4 C_{3}|I|^{2} .
\end{aligned}
$$

In case $p \leq 0<q$ we use the right inequality of Lemma 9 to obtain

$$
\begin{aligned}
\sum_{n \in I} d_{n} \sum_{n \in I} d_{n}^{-1} \leq & C_{3}\left[(2+q)^{1-2 \alpha}+(|p|+1)^{1-2 \alpha}\right]\left[(2+q)^{1+2 \alpha}+(|p|+1)^{1+2 \alpha}\right] \\
= & C_{3}\left[(2+q)^{2}+(1-p)^{1-2 \alpha}(2+q)^{1+2 \alpha}+(2+q)^{1-2 \alpha}(1-p)^{1+2 \alpha}\right. \\
& \left.+(1-p)^{2}\right] \leq 2 C_{3}\left[(2+q)^{2}+(1-p)^{2}\right] \leq 2 C_{3}(q-p+3)^{2} \\
\leq & 18 C_{3}(q-p+1)^{2}=18 C_{3}|I|^{2} .
\end{aligned}
$$

The remaining cases are similar.

The preceding lemma leads to a sufficient condition for complete interpolating sequences which is analogous to the following generalization of Theorem 3 .

Theorem 7. A sequence $\left\{\lambda_{n}\right\}_{n \in \mathbf{Z}} \subset \mathbf{R}$ is a complete interpolating sequence if conditions (i), (ii) of Theorem 2 hold as well as

$$
|F(x+\mathrm{i} y)| \asymp(1+|x|)^{\alpha}, \quad-\frac{1}{2}<\alpha<\frac{1}{2} .
$$

Pavlov [27] attributes this statement to a private communication of Katsnelson, and it is also contained in more general results by Avdonin [2] and Sedletskii [30]. [19] contains an example which shows that the theorem becomes false for $|\alpha| \geq \frac{1}{2}$. Of course, the case $\alpha=0$ reduces to Theorem 3 .

Theorem 6 and Lemma 5 imply the following discrete analogue.

Corollary 1. Let $s=\left\{c_{n}\right\}_{n \in \mathbf{Z}}$ be a sequence with $(-1)^{n} c_{n} \geq 0$ and

$$
\left|c_{n}\right| \asymp(1+|n|)^{\alpha}, \quad-\frac{1}{2}<\alpha<\frac{1}{2},
$$

and let $\varphi: \mathbf{C}_{-} \rightarrow \Omega(s)$ be a conformal map with $\lim _{y \rightarrow-\infty} \operatorname{Re}(\mathrm{i} y)=\infty$. If $\varphi$ is appropriately normalized then the entire function $F$ in (6) has exponential type $\pi$, and $\left\{\lambda_{n}\right\}_{n \in \mathbf{Z}}=F^{-1}(0)$ is a complete interpolating sequence.

It is not difficult to see that this statement becomes false for other values of $\alpha$ since also Lemma 5 fails in this case. 
Next we remark that the condition $\lim _{y \rightarrow-\infty} \operatorname{Re}(\mathrm{i} y)=\infty$ in Theorem 6 determines $\varphi$ up to conformal self-maps of $\mathbf{C}_{-}$that fix $\infty$. Hence $\varphi(a z+b), a>0, b \in \mathbf{R}$ describes the set of all conformal mappings of $\mathbf{C}_{-}$onto $\Omega(s)$ with this condition if $\varphi$ is one such mapping. Though we know that $F(z)=\mathrm{e}^{\varphi(a z+b)}$ has exponential type, it is not clear for which $a>0$ the type is equal to $\pi$. Obviously, the type does not depend on $b$, whereas it is a linear function of $a$. Hence there is exactly one $a>0$ such that the type of $F$ is equal to $\pi$. In order to indentify this value we introduce the upper and lower density of a sequence $\left\{\lambda_{n}\right\}_{n \in \mathbf{Z}}$ by

$$
D^{+}:=\lim _{r \rightarrow \infty} \max _{x \in \mathbf{R}} \frac{\left|\left\{\lambda_{n}\right\} \cap[x-r, x+r)\right|}{2 r}, \quad D^{-}:=\lim _{r \rightarrow \infty} \min _{x \in \mathbf{R}} \frac{\left|\left\{\lambda_{n}\right\} \cap[x-r, x+r)\right|}{2 r} .
$$

It follows from results of Landau [15] that $D^{+}=D^{-}=1$ is necessary (but not sufficient) for complete interpolating sequences. The densities of $F^{-1}(0)$, regarded as a function of $a$, are of the form const/a. Hence the density conditions are sufficient to identify the right conformal mapping.

Corollary 2. A sequence $\left\{\lambda_{n}\right\}_{n \in \mathbf{Z}} \subset \mathbf{R}$ is a complete interpolating sequence if and only if it is the zero set of $F$ in $(6)$, and $D^{+}=1\left(D^{-}=1\right)$, where $\varphi: \mathbf{C}_{-} \rightarrow \Omega(s)$ is a conformal map and $\left\{d_{n}\right\}_{n \in \mathbf{Z}}=\left\{c_{n}^{2}\right\}_{n \in \mathbf{Z}}$ satisfies $\left(\tilde{A}_{2}\right)$.

Now it becomes clear what was meant by a parameterization of the set of complete interpolating sequences by independent parameters. For any sequence $\left\{d_{n}\right\}_{n \in \mathbf{Z}}$ of positive real numbers satisfying $\left(\tilde{A}_{2}\right)$ there is a unique sequence $s=\left\{c_{n}\right\}_{n \in \mathbf{Z}}$ with $c_{n}^{2}=d_{n}$ and $(-1)^{n} c_{n} \geq 0$. Let $\varphi: \mathbf{C}_{-} \rightarrow \Omega(s)$ be a conformal map such that $D^{+}=D^{-}=1$ holds for $\left\{\lambda_{n}\right\}_{n \in \mathbf{Z}}:=\varphi^{-1}(-\infty)$. Then $\left\{\lambda_{n}\right\}_{n \in \mathbf{Z}}$ is uniquely determined up to shifts $\lambda \mapsto \lambda+b, b \in \mathbf{R}$. If we fix one value of the sequence $\left\{\lambda_{n}\right\}_{n \in \mathbf{Z}}$, this sequence is even unique. Conversely, if we start with a complete interpolating sequence $\left\{\lambda_{n}\right\}_{n \in \mathbf{Z}}$, the generating function and hence also the sequence of its critical values is unique up to multiplication by a positive constant. Thus we have proved

Corollary 3. Let $\lambda \in \mathbf{R}, d>0$. Theorem 6 describes a one-to-one correspondence between all positive sequences $\left\{d_{n}\right\}_{n \in \mathbf{R}}$ with $\left(\tilde{A}_{2}\right)$ and $d_{0}=d$, and all real complete interpolating sequences $\left\{\lambda_{n}\right\}_{n \in \mathbf{Z}}$ with $\lambda_{0}=\lambda$.

\section{Connection with the discrete Muckenhoupt condition for values of the derivative}

As mentioned in section 1, the discrete Muckenhoupt condition $\left(\tilde{A}_{2}\right)$ was studied by Lyubarskii and Seip [22] for the numbers $\left|F^{\prime}\left(\lambda_{n}\right)\right|^{2}$. It is intuitively clear that there is a connection between the values $\left\{c_{n}\right\}_{n \in \mathbf{Z}}$ of $F$ at its critical points and the magnitude of the derivative at the zeros of $F$. This will be made precise in the following lemma.

Lemma 6. Let $\left\{\lambda_{n}\right\}_{n \in \mathbf{Z}} \subset \mathbf{R}$ be a complete interpolating sequence, $F$ its generating function, and $\left\{c_{n}\right\}_{n \in \mathbf{Z}}$ its critical values. Then

$$
\left|F^{\prime}\left(\lambda_{n}\right)\right| \asymp\left|c_{n}\right|
$$


Proof. The proof relies on the same technique as Lemma 4. Subtract $\log \left|x-\lambda_{n}\right|$ on both sides of (26) and then let $x \rightarrow \lambda_{n}$. We obtain

$$
\begin{aligned}
\log \left|F^{\prime}\left(\lambda_{n}\right)\right|= & B|y|+\sum_{k \in \mathbf{Z} \backslash\{n\}} \frac{1}{2} \log \left(1-\frac{4 y^{2}}{\left(\lambda_{k}-\lambda_{n}\right)^{2}+4 y^{2}}\right)-\log (2|y|) \\
& +\frac{1}{\pi} \int_{\mathbf{R}} \frac{|y|}{\left|\lambda_{n}-\mathrm{i} y+t\right|^{2}} \log |F(t+\mathrm{i} y)| d t
\end{aligned}
$$

Using $\left|\lambda_{n}-\lambda_{k}\right| \geq \delta|n-k|$, where $\delta$ ist the separation constant from (3), we obtain

$$
\begin{aligned}
& |\log | F^{\prime}\left(\lambda_{n}\right)\left|-\frac{1}{\pi} \int_{\mathbf{R}} \frac{|y|}{\left|\lambda_{n}-\mathrm{i} y+t\right|^{2}} \log \right| F(t+\mathrm{i} y)|d t| \\
& \leq B|y|+|\log (2|y|)|-\sum_{k=1}^{\infty} \log \left(1-\frac{4 y^{2}}{k^{2} \delta^{2}+4 y^{2}}\right)=: C_{1} .
\end{aligned}
$$

If $I_{\tilde{x}_{n}} \subset\left[\lambda_{n-1}, \lambda_{n}\right]$ is again an interval of length $\delta$ centered at $\tilde{x}_{n}$, and $\psi(x)=$ $\log |F(x+\mathrm{i} y)|$, a similar computation as in Lemma 4 shows

$$
\left|\frac{1}{\pi} \int_{\mathbf{R}} \frac{|y|}{\left|\lambda_{n}-\mathrm{i} y+t\right|^{2}} \log \right| F(t+\mathrm{i} y)\left|d t-\psi_{I_{\tilde{x}_{n}}}\right| \leq C_{2}, \quad n \in \mathbf{Z}
$$

for a positive constant $C_{2}$ independent of $n$. From the estimate (32) in the proof of Lemma 4, (33), and (34) we get

$$
|\log | F^{\prime}\left(\lambda_{n}\right)|-\log | c_{n}|| \leq C_{22}+C_{1}+C_{2},
$$

hence the quotient $\left|F^{\prime}\left(\lambda_{n}\right)\right| /\left|c_{n}\right|$ lies between certain positive constants for all values of $n$.

We remark that it is not necessary for the preceding lemma that the critical value $c_{n}$ is assumed in the interval $\left(\lambda_{n-1}, \lambda_{n}\right)$. From (8) follows that $\left|c_{n}\right| \asymp\left|c_{n+N}\right|$ for every fixed value of $N$, therefore the critical points and zeros of $F$ only have to be both in ascending order.

Lemma 6 can be used to show a version of Pavlov's theorem involving the values $\left|F^{\prime}\left(\lambda_{n}\right)\right|$. In contrast to condition (iii') in section 1, every relatively dense subsequence $\left\{\lambda_{n_{k}}\right\}_{k \in \mathbf{Z}}$ can be considered, i.e. also the full sequence $\left\{\lambda_{n}\right\}_{n \in \mathbf{Z}}{ }^{3}$.

Corollary 4. A sequence $\left\{\lambda_{n}\right\}_{n \in \mathbf{Z}} \subset \mathbf{R}$ is a complete interpolating sequence if and only if conditions (i), (ii) of Theorem 2 hold as well as

(iii") For one (and then for every) relatively dense subsequence $\left\{\lambda_{n_{k}}\right\}_{k \in \mathbf{Z}}$ and the values $d_{k}:=\left|F^{\prime}\left(\lambda_{n_{k}}\right)\right|^{2}$ the discrete Muckenhoupt condition $\left(\tilde{A}_{2}\right)$ is true.

Proof. We will not reprove the sufficiency of (i),(ii),(iii") which is done in [22]. We only show the discrete Muckenhoupt condition for the sequence $\left\{d_{k}\right\}_{k \in \mathbf{Z}}$ if $\left\{\lambda_{n_{k}}\right\}_{k \in \mathbf{Z}}$ is any relatively dense subsequence. We can assume that $n_{k}<n_{k+1}$ for all $k \in \mathbf{Z}$. The relative density yields

$$
\Delta_{2}:=\sup _{k \in \mathbf{Z}}\left|\lambda_{n_{k}}-\lambda_{n_{k+1}}\right|<\infty
$$

and thus

$$
\delta\left|n_{p}-n_{q}\right| \leq\left|\lambda_{n_{p}}-\lambda_{n_{q}}\right| \leq|p-q| \Delta_{2}, \quad \forall p, q \in \mathbf{Z} .
$$

\footnotetext{
${ }^{3}$ It seems that the arguments in [22] show the same, only the formulation of the result is weaker.
} 
For any finite set $I=\{p, \ldots, q\}$ of consecutive integers, Theorem 6 and Lemma 6 imply

$$
\begin{aligned}
& \sum_{k \in I}\left|F^{\prime}\left(\lambda_{n_{k}}\right)\right|^{2} \sum_{k \in I}\left|F^{\prime}\left(\lambda_{n_{k}}\right)\right|^{-2} \leq C \sum_{k \in I} c_{n_{k}}^{2} \sum_{k \in I} c_{n_{k}}^{-2} \leq C \sum_{k=n_{p}}^{n_{q}} c_{k}^{2} \sum_{k=n_{p}}^{n_{q}} c_{k}^{-2} \\
& \leq C^{\prime}\left(n_{q}-n_{p}+1\right)^{2} \leq C^{\prime}\left((q-p) \frac{\Delta_{2}}{\delta}+1\right)^{2} \leq C^{\prime} \max \left(\frac{\Delta_{2}}{\delta}, 1\right)^{2}|I|^{2} .
\end{aligned}
$$

\section{Summary and open questions}

The main result of this paper is Theorem 6 which characterizes complete interpolating sequences. In contrast to known characterizations of such sequences, the separation of the points and the exponential type of the generating function follow automatically. The relation with conformal mappings makes the application of distortion theorems possible, and the discrete version $\left(\tilde{A}_{2}\right)$ of the Muckenhoupt condition $\left(A_{2}\right)$ is easier to verify for concrete applications. Unfortunately, our approach does not give an independent proof of these characterizations, but is based on a comparison argument with the classical Theorem 2. For this reason, one can expect to extend this argument to known generalizations of Pavlov's Theorem, for example the one given in [22] for Paley-Wiener spaces $P W_{\pi}^{p}$.

An entire function of exponential type at most $\pi$ is said to be in $P W_{\pi}^{p}, 1<p<\infty$, if it belongs to $L^{p}$ on the real line, and a sequence $\left\{\lambda_{n}\right\}_{n \in \mathbf{Z}}$ is called complete interpolating sequence for $P W_{\pi}^{p}$ if for every sequence $\left\{a_{n}\right\}_{n \in \mathbf{Z}}$ with (1) the interpolation problem (2) has a unique solution $f \in P W_{\pi}^{p}$. It is straightforward to see that a characterization analogous to Theorem 6 of real complete interpolating sequences is valid, if we impose the discrete Muckenhoupt condition

$$
\sum_{n \in I} d_{n} \sum_{n \in I} d_{n}^{-1 /(p-1)} \leq C|I|^{p}
$$

on the sequence $\left\{d_{n}\right\}_{n \in \mathbf{Z}}=\left\{\left|c_{n}\right|^{p}\right\}_{n \in \mathbf{Z}}$.

On the other hand, the restriction to real sequences can not so easily be disposed of. As long as the imaginary parts of $\left\{\lambda_{n}\right\}_{n \in \mathbf{Z}}$ are bounded we can refer to Corollary 1 in section 8 of Chapter 4 in [34] which asserts that $\left\{\mathrm{e}^{\operatorname{Re} \lambda_{n} i t}\right\}_{n \in \mathbf{Z}}$ is a Riesz basis in $L^{2}(-\pi, \pi)$ if and only if $\left\{\mathrm{e}^{\lambda_{n} i t}\right\}_{n \in \mathbf{Z}}$ is so. It would be very interesting to know if a representation by conformal mappings of the generating function can also be found in the general case, where the reflection principle does not work immediately.

\section{Lemmas}

In this section we give the proofs of some lemmas that had been postponed in the main text.

Lemma 7. For the module $s\left(r_{1}, r_{2}\right)$ of the family of curves connecting the noncircular sides of $S\left(r_{1}, r_{2}\right)$ defined in (15) we have (17).

Proof. The principal value of the logarithm maps $S\left(r_{1}, r_{2}\right)$ conformally onto

$$
\tilde{S}\left(r_{1}, r_{2}\right):=\left\{x+\mathrm{i} y: \log r_{1}<x<\log r_{2}, \varphi_{2}(x)<y<\varphi_{1}(x)\right\},
$$

where $\varphi_{1}(x):=\frac{\pi}{2}\left(1-C_{4} x \mathrm{e}^{-x}\right)$ and $\varphi_{2}(x):=-\varphi_{1}(x)$, see Figure 3 . 

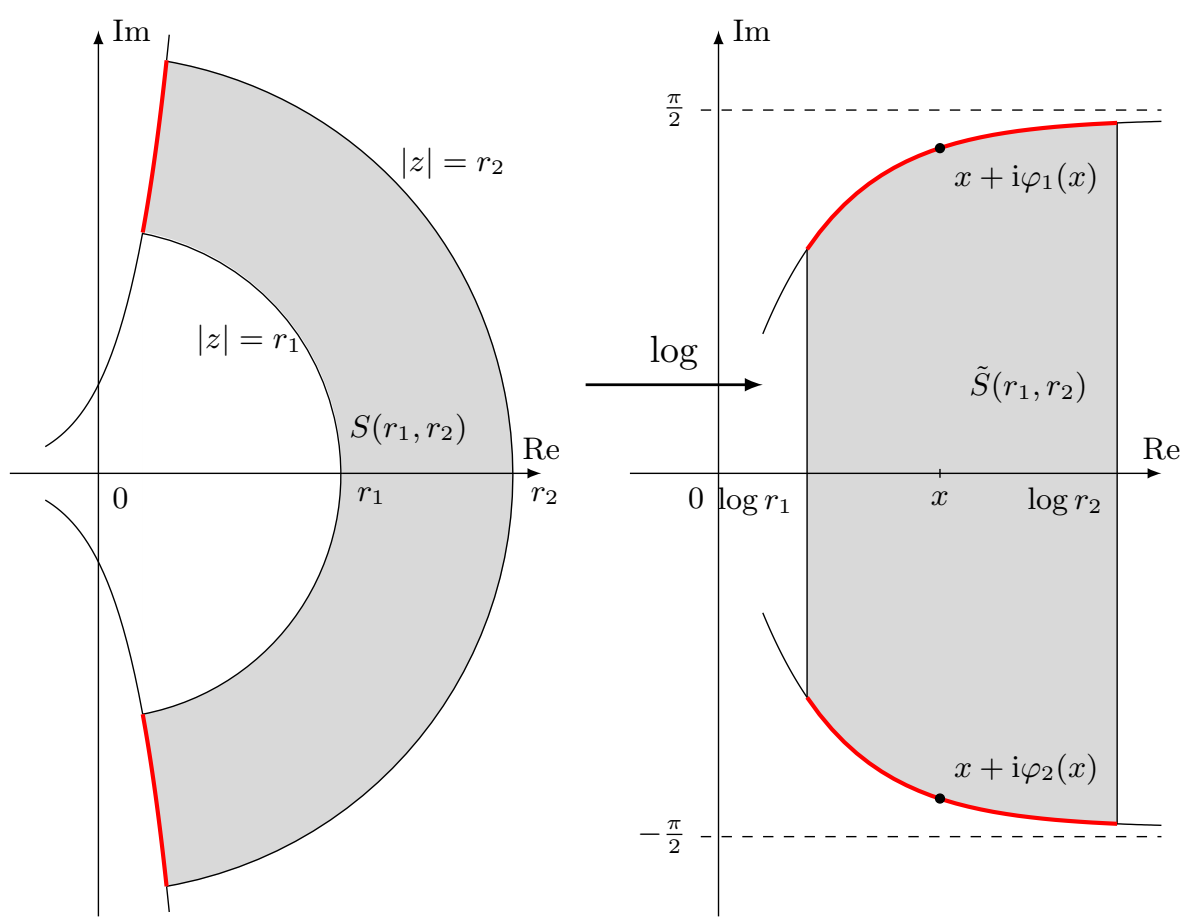

Figure 3. Conformal mapping of $S\left(r_{1}, r_{2}\right)$ onto $\tilde{S}\left(r_{1}, r_{2}\right)$.

By the conformal invariance of the module we know that

$$
s\left(r_{1}, r_{2}\right)=\tilde{s}\left(r_{1}, r_{2}\right),
$$

where $\tilde{s}\left(r_{1}, r_{2}\right)$ is the module of the family of all curves joining $\left\{x+\mathrm{i} \varphi_{1}(x): \log r_{1} \leq\right.$ $\left.x \leq \log r_{2}\right\}$ and $\left\{x+\mathrm{i} \varphi_{2}(x): \log r_{1} \leq x \leq \log r_{2}\right\}$ in $\tilde{S}\left(r_{1}, r_{2}\right)$. Denoting $\vartheta(x):=$ $\varphi_{1}(x)-\varphi_{2}(x)=\pi\left(1-C_{4} x \mathrm{e}^{-x}\right)$ and applying formula (13.4) from [29] we get the estimate

$$
\int_{\log r_{1}}^{\log r_{2}} \frac{d x}{\vartheta(x)} \leq \tilde{s}\left(r_{1}, r_{2}\right) \leq \int_{\log r_{1}}^{\log r_{2}} \frac{d x}{\vartheta(x)}+R\left(r_{1}, r_{2}\right)
$$

where

$$
0 \leq R\left(r_{1}, r_{2}\right) \leq \int_{\log r_{1}}^{\log r_{2}} \frac{\varphi_{1}^{\prime}(x)^{2}+\varphi_{2}^{\prime}(x)^{2}}{\vartheta(x)} d x
$$

We compute

$$
\begin{aligned}
\left|\int_{\log r_{1}}^{\log r_{2}} \frac{d x}{\vartheta(x)}-\frac{1}{\pi} \log \frac{r_{2}}{r_{1}}\right| & =\left|\frac{1}{\pi} \int_{\log r_{1}}^{\log r_{2}} \frac{d x}{1-C_{4} x \mathrm{e}^{-x}}-\frac{1}{\pi} \int_{\log r_{1}}^{\log r_{2}} d x\right| \\
& =\frac{1}{\pi} \int_{\log r_{1}}^{\log r_{2}} \frac{C_{4} x \mathrm{e}^{-x}}{1-C_{4} x \mathrm{e}^{-x}} d x \rightarrow 0
\end{aligned}
$$

as $r_{1}, r_{2} \rightarrow \infty$. On the other hand, (38) yields

$$
R\left(r_{1}, r_{2}\right) \rightarrow 0 \quad \text { as } r_{1}, r_{2} \rightarrow \infty,
$$


since $\int_{x_{0}}^{\infty} \frac{\varphi_{1}^{\prime}(x)^{2}+\varphi_{2}^{\prime}(x)^{2}}{\vartheta(x)} d x$ is readily seen to converge. Now (36), (37), (39) and (40) imply the assertion (17).

Let $\bmod \left(E_{1}, E_{2}\right)$ denote the module of the family of curves connecting two sets $E_{1}, E_{2}$ in $\mathbf{C}_{-}$. Then we have the following lemma.

Lemma 8. For every $\varepsilon>0$ there is $\delta \in(0,1)$ with the property that for all $-1<a<b<1$ with $\varepsilon<\bmod ([a, b],[1, \infty))<1 / \varepsilon$ and $\varepsilon<\bmod ([a, b],(-\infty,-1])<$ $1 / \varepsilon$ we have $|a|,|b| \leq \delta$.

Proof. As in [1] we denote by $\Lambda(R)$ the extremal distance of $[-1,0]$ from $[R, \infty)$ in $\mathbf{C}$. The module of all curves joining $[-1,0]$ and $[R, \infty)$ in $\mathbf{C}_{-}$is then $1 /(2 \Lambda(R))$, and we get

$$
\bmod ([a, b],[1, \infty))=\frac{1}{2 \Lambda\left(\frac{1-b}{b-a}\right)}, \quad \bmod ([a, b],(-\infty,-1])=\frac{1}{2 \Lambda\left(\frac{1+a}{b-a}\right)} .
$$

Since $\Lambda(R)$ is monotonical, tends to $+\infty$ as $R \rightarrow+\infty$, and to 0 as $R \rightarrow 0$, our assumptions imply that there are constants $C, c>0$ depending only on $\varepsilon$ such that

$$
c \leq \frac{1-b}{b-a} \leq C, \quad c \leq \frac{1+a}{b-a} \leq C .
$$

Adding both inequalities yields after elementary manipulations

Hence

$$
b-a \geq \frac{2}{2 C+1} .
$$

$$
1-b \geq \frac{2 c}{2 C+1}, \quad a+1 \geq \frac{2 c}{2 C+1},
$$

and the assertion is true if we put $\delta:=1-(2 c) /(2 C+1)$.

Lemma 9. For real numbers $p, q, \alpha$ with $p, q>0$ and $-\frac{1}{2} \leq \alpha \leq \frac{1}{2}$ holds

$$
2 p q \leq p^{1+2 \alpha} q^{1-2 \alpha}+p^{1-2 \alpha} q^{1+2 \alpha} \leq p^{2}+q^{2} .
$$

Proof. We fix $p$ and $q$ and consider the function $g(\alpha):=p^{1+2 \alpha} q^{1-2 \alpha}+p^{1-2 \alpha} q^{1+2 \alpha}$. We have $g(0)=2 p q, g\left(\frac{1}{2}\right)=p^{2}+q^{2}$, and computation of $g^{\prime}(\alpha)$ shows that $g(\alpha)$ grows monotonically on $\left[0, \frac{1}{2}\right]$. For negative $\alpha$ the result follows from $g(\alpha)=g(-\alpha)$.

An elementary proof can be given using the arithmetic-geometric means inequality for the left estimate and the rearrangement inequality [12] for the right estimate. The statement is also contained in Muirhead's inequality [12].

Acknowledgement. I thank A. E. Eremenko for a discussion of [6] and many useful suggestions. MATLAB and the Schwarz-Christoffel toolbox by T. Driscoll [5] were helpful in the creation of the figures.

\section{References}

[1] Ahlfors, L. V.: Conformal invariants: topics in geometric function theory. - McGraw-Hill, 1973.

[2] Avdonin, S. A.: On the question of Riesz bases of exponentials in $L^{2}$. - Vestn. Leningr. Univ. Ser. Mat. 13, 1974, 5-12; English transl. in Vestn. Leningr. Univ. Math. 7, 1979, 203-211. 
[3] Avdonin, S. A., and I. Joó: Riesz bases of exponentials and sine-type functions. - Acta Math. Hung. 51, 1988, 3-14.

[4] Christensen, O.: An introduction to frames and Riesz bases. - Birkhäuser, Boston, 2003.

[5] Driscoll, T.: The Schwarz-Christoffel toolbox. - http://www.math.udel.edu/ driscoll/software/.

[6] Eremenko, A. E., and M. L. Sodin: Paramterization of entire functions of sine-type by their critical values. - Adv. Sov. Math. 11, 1992, 237-242.

[7] Garnett, J. B.: Bounded analytic functions. - Academic Press New York, 1981.

[8] Garnett, J. B., and D. E. Marshall: Harmonic measure. - Cambridge University Press, 2005.

[9] Gohberg, I. C., and M. G. Krein: Introduction to the theory of linear nonselfadjoint operators. - Transl. Math. Monogr., American Mathematical Society, Providence, 1969.

[10] Goldberg, A. A.: On ramified values of entire functions. - Sibirsk. Mat. Zh. 14:4, 1973, 862-866; English transl. in Siberian Math. J. 14, 1973, 599-602.

[11] Golovin, V.D.: Biorthogonal expansions in linear combinations of exponential functions in $L_{2}$. - Zap. Mekh. Mat. Fak. Kharkov. Gos. Univ. i Kharkov. Mat. Obshch. 30:4, 1964, 18-29.

[12] Hardy, G. H., J. E. Littlewood, and G. Pólya: Inequalities. - Cambridge University Press, 1934.

[13] Kadets, M. I.: The exact value of the Paley-Wiener constant. - Sov. Math. Dokl. 5, 1964, $559-561$.

[14] Koosis, P.: The logarithmic integral I. - Cambridge University Press, 1988.

[15] Landau, H. J.: Necessary density conditions for sampling and interpolation of certain entire functions. - Acta Math. 117, 1967, 37-52.

[16] Lawrentjew, M.A., and B. W. Schabat: Methoden der komplexen Funktionentheorie. VEB Deutscher Verlag der Wissenschaften, 1967.

[17] Levin, B. YA.: On bases of exponential functions in $L_{2}$. - Zap. Mekh. Mat. Fak. Kharkov. Gos. Univ. i Kharkov. Mat. Obshch. 27:4, 1961, 39-48.

[18] Levin, B. YA.: Interpolation by entire functions of exponential type. - Mat. Fiz. i Funktsional. Anal. 1, 1969, 136-146.

[19] Levin, B. YA.: Lectures on entire functions. - Transl. Math. Monogr. 150, AMS, 1996.

[20] Levin, B. YA., and I. V. Ostrovskir: On small perturbations of the set of zeros of functions of sine type. - Izv. Akad. Nauk SSSR Ser. Mat. 43:1, 1979, 87-110; English transl. in Math. USSR Izv. 14:1, 1980, 79-101.

[21] Levin, B. YA., and I. V. OstrovskiI: Zero sets of sine-type functions. - J. Sov. Math. 26:5, 1984, 2287-2288.

[22] LyubarskiI, Yu. I., and K. SeiP: Complete interpolating sequences for Paley-Wiener spaces and Muckenhoupt's $\left(A_{p}\right)$ condition. - Rev. Mat. Iberoamericana 13:2, 1997, 361-376.

[23] Minkin, A. M.: Reflection of exponents, and unconditional bases of exponentials. - Algebra Anal. 3:5, 1991, 109-134, English transl. in St. Petersburg Math. J. 3:5, 1992, 1043-1068.

[24] Nikol'skiI, N. K.: Bases of exponentials and the values of reproducing kernels. - Dokl. Akad. Nauk SSSR 252, 1980, 1316-1320; English transl. in Sov. Math. Dokl. 21, 1980, 937-941.

[25] Paley, R. E. A. C., and N. Wiener: Fourier transforms in the complex domain. - Amer. Math. Soc. Colloq. Publ. 19, American Mathematical Society, New York, 1934.

[26] Partington, J.R.: Interpolation, identification, and sampling. - Oxford University Press, 1997. 
[27] Pavlov, B. S.: Basicity of an exponential system and Muckenhoupt's condition. - Dokl. Akad. Nauk SSSR 247:1, 1979, 37-40; English translation in Sov. Math. Dokl. 20:4, 1979, 655-659.

[28] Pommerenke, Ch.: Boundary behaviour of conformal maps. - Grundlehren Math. Wiss. 299, Springer-Verlag, 1992.

[29] Rodin, B., and S. E. Warschawski: Extremal length and the boundary behavior of conformal mappings. - Ann. Acad. Sci. Fenn. Ser. A I Math. 2, 1976, 467-500.

[30] Sedletskit, A. M.: Expansions in exponential functions. - Sibirsk. Mat. Zh. 16:4, 1975, 820829; English transl. in Siberian Math. J. 16, 1975, 628-635.

[31] Sedletskit, A. M.: Biorthogonal expansions of functions in series of exponents on intervals of the real axis. - Usp. Mat. Nauk 37:5, 1982, 51-95; English transl. in Russ. Math. Surv. 37:5, $1982,57-108$.

[32] SedLetskiI, A. M.: Fourier transforms and approximations. - Analytical methods and special functions, Gordon and Breach Science Publishers, 2000.

[33] Tsuji, M.: Potential theory in modern function theory. - Chelsea Publishing Company, 1975.

[34] Young, R. M.: An introduction to nonharmonic Fourier series. - Academic Press, New York, 1980.

Received 2 April 2008 